\title{
A Mathematical Model of the Human Metabolic System and Metabolic Flexibility
}

\author{
T. Pearson • J.A.D. Wattis • J.R. King •
}

I.A. MacDonald • D.J. Mazzatti

Received: date / Accepted: date

\begin{abstract}
In healthy subjects some tissues in the human body display metabolic flexibility, by this we mean the ability for the tissue to switch its fuel source between predominantly carbohydrates in the post prandial state and predominantly fats in the fasted state. Many of the pathways involved with human metabolism are controlled by insulin, and insulin-resistant states such as obesity and type- 2 diabetes are characterised by a loss or impairment of metabolic flexibility.

In this paper we derive a system of 12 first-order coupled differential equations that describe the transport between and storage in different tissues of the human body. We find steady state solutions to these equations and use these results to nondimensionalise the model. We then solve the model numerically to simulate a healthy balanced meal and a high fat meal and we discuss and compare these results. Our numerical results show good agreement with experimental data where we have data available to us and the results show behaviour that agrees with intuition where we currently have no data with which to compare.
\end{abstract}

Keywords Multicompartmental Modelling · Insulin · Glucose · Free Fatty Acids - Triglyceride

T. Pearson (sadly died in March 2013, following a long illness)

Centre for Mathematical Medicine and Biology, School of Mathematical Sciences, University of Nottingham, University Park, Nottingham, NG7 2RD, UK.

J.A.D. Wattis (corresponding author)

Centre for Mathematical Medicine and Biology, School of Mathematical Sciences, University of Nottingham, University Park, Nottingham, NG7 2RD, UK.

J.R. King

Centre for Mathematical Medicine and Biology, School of Mathematical Sciences, University of Nottingham, University Park, Nottingham, NG7 2RD, UK.

I.A. MacDonald

The University of Nottingham Medical School, Queen's Medical Centre, Nottingham, NG7 2UH, UK.

D.J. Mazzatti

Unilever Discover, Colworth Science Park, Sharnbrook, Bedfordshire, MK44 1LQ, UK. 


\section{Introduction}

The human diet consists of a mix of carbohydrates and fats delivered in a discontinuous manner whilst the human body is constantly expending energy. To deal with this discontinuous supply of energy from the diet, human physiology has evolved mechanisms to manage the transport, storage and utilisation of carbohydrates and fats between the different tissues of the body.

In lean healthy subjects skeletal muscle displays metabolic flexibility, by this we mean that the body is able to switch its fuel source between predominantly carbohydrates in the post prandial, insulin stimulated, state and predominantly fats in the fasted state. The main regulatory hormone involved in metabolism is insulin, and because of this, insulin resistant states such as type 2 diabetes and obesity are characterised by this loss of metabolic flexibility $[1,2]$.

Insulin resistance or insulin deficient states can be generalised into three categories: (i) abnormal $\beta$-cell secretory product, resulting in lower levels of insulin being produced by the $\beta$-cells of the pancreas, that is, insulin insufficiency; (ii) insulin antagonists in the blood plasma, either due to counterregulatory hormones or due to non-hormonal bodies that either affect insulin receptors or alter insulin signalling effectiveness; (iii) the target tissue being defective in insulin action, due either to insulin receptor defects or to defects in the effector systems. The third of these causes is of most interest to us as this form of insulin resistance is common in some obese and all type 2 diabetic subjects, although early phase insulin secretion is inhibited in some type 2 diabetic subjects [3,4]. Both obese and diabetic subjects demonstrate a reduced rate of plasma glucose disposal and a higher than normal basal glucose level [3-5]. In the case of type 2 diabetes this can be partially explained by the fact that hepatic glucose uptake is impaired $[4,6]$ and the suppression of hepatic glucose output by insulin is reduced [7]; also skeletal muscle glucose uptake is known to be impaired in type 2 diabetes [8]. Similarly, with non-diabetic obese subjects there is reduced hepatic glucose output suppression from insulin [3,5] and reduced skeletal muscle glucose uptake [9]. It has also been shown that insulin resistant subjects can have increased hepatic lipogenesis, resulting in larger changes in hepatic triglyceride concentrations in the post prandial state which is counter intuitive as it is an insulin stimulated pathway; however, it was also shown that the insulin resistant subjects had a much greater reduction in muscle glucose uptake indicating that insulin resistance affects different tissues to different degrees [10] with the liver being less severely affected [11]. It is not just glucose metabolism that is affected by insulin resistance, it also affects adipose tissue with reduced insulin suppression of free fatty acid release common in obesity [9]. Insulin resistant subjects also show reduced adipose tissue triglyceride clearance and reduced skeletal muscle triglyceride clearance [13]. Fatty liver disease is a large area of interest currently as it is closely related to obesity and insulin resistance. Although it has been shown that there is a strong correlation between obesity and fatty liver disease [14] it is not known if fatty liver disease is the cause or effect of insulin resistance. It has been shown that post-prandial hepatic triglyceride release is higher in people with fatty liver disease than healthy people although the basal secretion rate is the same, indicating that it is not a higher fat presence in the liver which causes the increased output but rather insulin resistance [15]. 
Although there has been much work published on mathematical modelling of human metabolism much has focused on single tissues or a limited number of substrates and, in some cases, on metabolism at a cellular level. Glucose-insulin metabolism has been extensively investigated with many models describing the effect of insulin supply on glucose metabolism [22-25] and conversely the effects of a controlled glucose supply on insulin dynamics [26]. More complex models have also been proposed $[27-29,43]$ which although detailed are still limited to glucoseinsulin metabolism. There are also models focusing on the metabolism of a variety of lipoproteins in the body and the transport of and conversion between them [30]. Other models describe specific types of lipoprotein and their competitive uptake in the liver [31-33]. Models of skeletal muscle metabolism, particularly in terms of energy balance, have also been proposed [34,35] however these are restricted to skeletal muscle and ignore the rest of the body. Some very detailed although very computational models have also been proposed covering skeletal muscle $[46,47]$ and whole body metabolism [44]. There has also been simpler, more analytical, models proposed to describe whole body metabolism of carbohydrates, fats and proteins on long time scales although these tend to focus on energy balance and weight gain [36-42]. A recent $\mathrm{PhD}$ thesis developed four detailed, separate, models of metabolism covering glucose-fat interactions, hepatic glucose metabolism, postprandial lipid metabolism and adipose tissue distribution [45].

In this paper we develop a compartmental mathematical model to describe the transport, storage and utilisation of glucose and fats in the human body covering many of the different tissues. In particular we pay close attention to the blood plasma, the liver and skeletal muscle and we include terms which model the rest of the tissues in the human body. We aim to produce a model describing whole body metabolism as opposed to more detailed models which focus on a single metabolic subsystem. As such we take a more simplified approach to modelling the complex physiological process in the human body but still retain enough detail to produce meaningful results. Once we have formulated our model in section 2 ; in section 3 we find steady state equations in the fasted state where steady concentrations are applicable, and use these to nondimensionalise the model, details of which are given in Appendix B. In section 4 we refer to the experimental literature to derive parameter values for the model. Finally we generate numerical results to the system of equations subject to realistic simulated meals in section 5 and in section 6 we draw conclusions.

\section{Mathematical Model of Metabolism}

In this section we formulate a mathematical model describing human metabolism and the dynamics of metabolic flexibility. We take account of the substrates glucose, glycogen, triglyceride (TAG), free fatty acids (FFA) and insulin in multiple compartments, of blood plasma, liver and skeletal muscle and we assume adipose tissue to be an unlimited source of FFA. Since we are concerned with how concentrations evolve over a 12-14 hour period this is quite reasonable. We model the synthesis and transport between compartments of each substrate. We assume the usage of glucose by the tissues and organs not modelled to be linearly dependent on the amount of each substrate in the blood plasma and that fat usage by the rest of the body is negligible. We derive the model one compartment at a time 


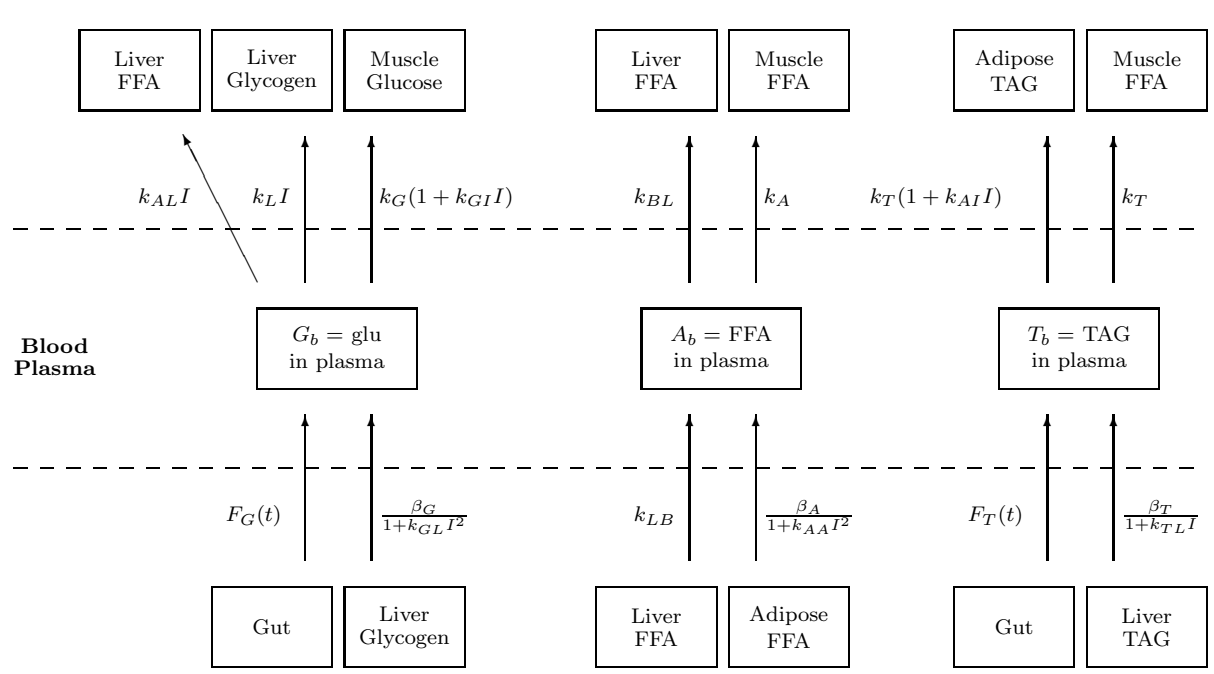

Fig. 1 Diagram showing the input and transportation of glucose, FFA and TAG in the blood plasma, not including utilisation by the rest of the body, with the rate of flux for each pathway shown.

and start with the blood plasma as this acts as the main transport medium for substrates between the different organs and tissues of the body and no synthesis of glucose, FFA or TAG occurs in the plasma.

Following ingestion of a meal, glucose and TAG are absorbed from the gut into the blood plasma and we will use this as the input for our model. As well as this absorption from the gut, FFA are released, at an insulin-inhibited rate, into the blood plasma from stores in adipose tissue. The presence of glucose and, to a lesser extent, FFA in the plasma stimulates the pancreas to produce and secrete insulin. We assume that insulin secretion dependence on FFA is small and model it by a linear dependence. Experimental data showing pancreatic insulin secretion rate compared to plasma glucose concentration [16] suggests a Hill function models the glucose-dependence. Whilst glucose concentrations at the higher end of the data are only observed, in vivo, in cases of hyperglycemia, we include the saturating effect whereby the insulin production term has some maximum value even at extreme glucose levels. Hence, with these assumptions we arrive at our first equation, modelling plasma insulin concentration by

$$
V_{b} \frac{d I}{d t}=k_{1}+k_{2} \operatorname{erf}\left(\left(G_{b}-v\right) / c\right)+k_{I A} A_{b}-\lambda_{I} I
$$

where the first two terms represent the saturating dependence on plasma glucose $\left(G_{b}\right)$, the third term represents the linear dependence on plasma FFA $\left(A_{b}\right)$ and the final term represents the linear decay of plasma insulin.

We now turn our attention to the uptake from and secretion to the plasma of glucose, TAG and FFA. The pathways we will now discuss are shown in figure 1. Glucose in the plasma may be taken up into skeletal muscle via two glucose transporters, the GLUT1 insulin-independent and GLUT4 insulin-dependent transporters. Other tissues of the body, such as the brain and the kidneys, use glucose as their energy source; we model this effect with the usage term $S_{G} G_{b}$ in 
the equations below. Glucose can also be taken up into and secreted by the liver, the uptake into the intracellular compartments of the liver, and the release back into the plasma, is handled by the GLUT2 insulin independent transporter and as such is entirely driven by concentration gradients. For this reason we make a simplification that we can ignore this step and instead we take account of what can happen to hepatic intracellular glucose. The storage of glucose in the liver as glycogen is mediated by insulin-stimulated enzymes and the liver has a finite storage capacity. The breakdown of glycogen back into glucose is mediated by insulin-inhibited enzymes. As well as storage as glycogen, glucose in the liver can be used to synthesise FFA in the liver via the pathways of glycolysis and subsequently lipogenesis, there are many steps in these pathways; however, we make use of the fact that each step in the pathways is insulin-stimulated and simplify this to a single insulin-stimulated step. It is worth noting that no glucose released from the breakdown of glycogen is used in FFA synthesis as the the breakdown of glycogen happens predominantly in the fasted, low insulin state, when the insulinstimulated pathway of hepatic FFA synthesis will be inactive, allowing our earlier simplification of ignoring intracellular glucose to remain valid. FFA in the plasma may be taken up into the liver and released back as well as being taken up into skeletal muscle and is handled by an insulin-independent fatty acid transporter.

As well as being absorbed from the gut TAG can be released into the plasma from the liver, this release is inhibited by insulin and is somewhat dependent on liver fat content, in that at lower liver fat levels secretion is reduced; however this secretion rate does not increase drastically at high liver fat levels. TAG in the plasma can be taken up into adipose tissue and skeletal muscle. Although TAG molecules are too large to be taken across the cell membrane they are bound to the cell walls and broken down by the enzyme lipoprotein lipase into FFA before being taken up into the cell. Lipoprotein lipase is thought to be dependent on insulin in adipose tissue and so we model the breakdown and uptake of TAG on this pathway as being both insulin-independent and insulin-dependent, however lipoprotein lipase is not insulin stimulated in skeletal muscle. Using these assumptions we arrive at the equations

$$
\begin{aligned}
V_{b} \frac{d G_{b}}{d t}= & \left(\frac{\beta_{G}}{1+k_{G L} I^{2}}\right) f_{1}\left(Y_{L}\right)-S_{G} G_{b}-k_{G}\left(1+k_{G I} I\right) G_{b}-k_{L} I G_{b} f_{2}\left(Y_{L}\right) \\
& -k_{A L} I G_{b}+F_{G}(t), \\
V_{b} \frac{d T_{b}}{d t}= & \left(\frac{\beta_{T}}{1+k_{T L} I}\right) f_{3}\left(T_{L}\right)-k_{T} T_{b}-k_{T A}\left(1+k_{A I} I\right) T_{b}+F_{T}(t), \\
V_{b} \frac{d A_{b}}{d t}= & \frac{\beta_{A}}{1+k_{A A} I^{2}}-k_{A} A_{b}-k_{B L} A_{b}+k_{L B} A_{L} .
\end{aligned}
$$

Equations (2)-(4) describe the concentrations of plasma glucose, TAG and FFA respectively where $Y_{L}, T_{L}$ and $A_{L}$ denote the concentrations of hepatic glucose (stored as glycogen), TAG and FFA respectively. We have introduced the dimensional parameter $V_{b}=$ blood volume. The nondimensional functions $f_{1}, f_{2}$ and $f_{3}$ are introduced so that the uptake and release of glucose and the release of TAG from the liver is dependent on how much is in the liver. In the case of $f_{1}$ the rate of release of glucose from the liver should depend on insulin with the main dependence on hepatic glycogen being that the release should be slow or switched 


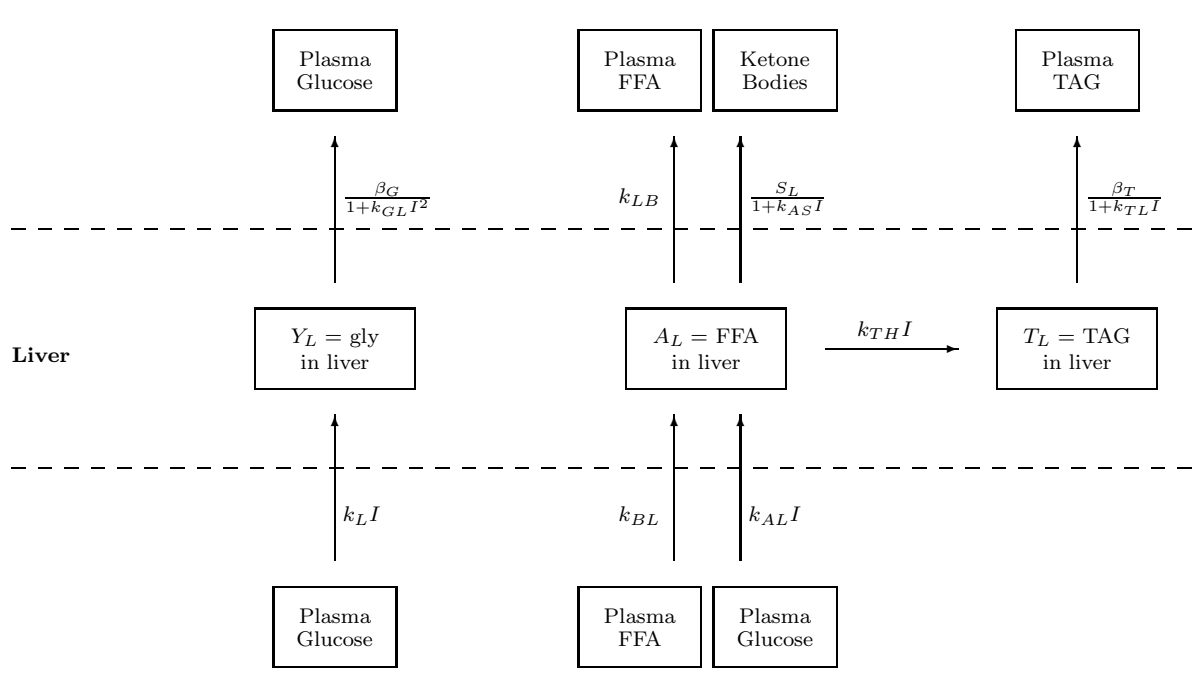

Fig. 2 Diagram showing the input and transportation of glucose, FFA and TAG in the liver with the rate of flux for each pathway shown.

off when the store is almost empty. Similar properties hold for the hepatic TAG release and the function $f_{3}$. In the case of $f_{2}$ the uptake of glucose by the liver should be mostly dependent on plasma glucose and insulin with the dependence on hepatic glycogen to slow or shut off completely the uptake when the liver is almost full. Hence we define these functions by

$$
f_{1}\left(Y_{L}\right)=\frac{Y_{L}}{Y_{0}+Y_{L}}, \quad f_{2}\left(Y_{L}\right)=\frac{Y_{\max }-Y_{L}}{Y_{0}+Y_{\max }-Y_{L}}, \quad f_{3}\left(T_{L}\right)=\frac{T_{L}}{T_{0}+T_{L}},
$$

where $Y_{\max }$ denotes the maximum amount of glycogen the liver can store and $Y_{0}$ and $T_{0}$ are small parameters. The final terms in equations (2) and (3) model the glucose and TAG absorbed by the gut following ingestion of a meal and are given by

$$
F_{G}(t)=\frac{\theta_{G} t}{B_{G}^{2}} e^{-t^{2} / 2 B_{G}^{2}}, \quad F_{T}(t)=\frac{\theta_{T} t}{B_{T}^{2}} e^{-t^{2} / 2 B_{T}^{2}} .
$$

These will act as our control inputs for the model and we are free to choose the parameters in these functions, $\theta_{G}$ and $\theta_{T}$ control the magnitude of the substrates entering the blood stream and $B_{G}$ and $B_{T}$ control the timescale for the release into the blood.

We now derive the equations for the liver, the pathways we discuss are shown in figure 2. As mentioned before, glucose from the plasma can be taken up into the liver and either stored as glycogen or used to synthesise fatty acids. Fatty acids from the plasma contribute to the hepatic FFA pool, with insulin independent uptake to and release from the liver. This FFA can be converted to fatty acetylCoA, via an insulin-independent process which is, in turn, either converted to TAG and stored in the liver, or oxidised to create ketone bodies. We make a simplification here in that we do not take account of the concentration of fatty acetyl-CoA but rather we will introduce two sink terms into the equation for hepatic FFA, to represent the FFA oxidised into ketone bodies and that stored as TAG. The storage as TAG is driven by an insulin-stimulated pathway and 
the oxidation to ketone bodies is primarily stimulated by glucagon. Currently our model does not include glucagon; however, it has been shown that glucagon and insulin are in general inversely related such that at high levels of insulin there is a low level of glucagon and vice versa [17, Ch. 5]. Hence we model this glucagonstimulation as insulin inhibition. As mentioned before, TAG stored in the liver is released back into the plasma at an insulin-inhibited rate. Using these assumptions we arrive at the equations

$$
\begin{aligned}
V_{l} \frac{d Y_{L}}{d t} & =k_{L} I G_{b} f_{2}\left(Y_{L}\right)-\left(\frac{\beta_{G}}{1+k_{G L} I^{2}}\right) f_{1}\left(Y_{L}\right) \\
V_{l} \frac{d A_{L}}{d t} & =k_{A L} I G_{b}-\frac{S_{L} A_{L}}{1+k_{A S} I}+k_{B L} A_{b}-k_{L B} A_{L}-k_{T H} I A_{L} \\
V_{l} \frac{d T_{L}}{d t} & =k_{T H} I A_{L}-\left(\frac{\beta_{T}}{1+k_{T L} I}\right) f_{3}\left(T_{L}\right) .
\end{aligned}
$$

Equations (7)-(9) describe the concentrations of hepatic glucose (stored as glycogen), FFA and TAG respectively. As with the equations for plasma concentrations (2)-(4) we have introduced the dimensional parameter $V_{l}=$ liver volume. We use the same units for our glycogen concentration variables as for glucose; thus glycogen is measured in effective, or equivalent, amount of glucose that can be stored in that form.

We complete the compartmental model by deriving equations for skeletal muscle. As well as modelling the uptake, storage and usage of glucose and FFA by the skeletal muscle we take account of energy expenditure since in skeletal muscle this has a large effect on the metabolism in the skeletal muscle. To model skeletal muscle energy expenditure we define a new variable, $P$, which is related to skeletal muscle AMP concentration; although not actually AMP concentration, it acts as a measure of flux through the skeletal muscle oxidative pathways. We assume that $P$ decays linearly and is decreased by oxidation with glucose or FFA in the skeletal muscle to create ATP, with glucose oxidation being insulin-stimulated and FFA oxidation insulin-independent. Using these assumptions we derive

$$
V_{s} \frac{d P}{d t}=\mu-\lambda_{P} P-\gamma_{a} M_{A} A_{m} P-\gamma_{g} M_{G} P I G_{m}
$$

The parameter $\mu$ acts as our measure of energy expenditure and is a control input for our model. As a first approximation we could assume a constant production of AMP from ATP, however, this is unphysical, as there is only a limited amount of ATP, ADP, and AMP available, so as AMP rises, ATP decreases, and the rate of production of AMP would also decrease. This effect is modelled in a simplistic way by the combined effect of $\mu$ and $\lambda$. The nondimensional parameters $\gamma_{a}$ and $\gamma_{g}$ are the number of $P$ molecules used in FFA and glucose oxidation respectively. The dimensional parameter $V_{s}=$ skeletal muscle volume.

We now derive equations for skeletal muscle glucose, glycogen, FFA and TAG. As before the pathways we will consider are shown in figure 3. As mentioned earlier, glucose is taken up from the plasma at insulin-stimulated and insulinindependent rates, FFA is taken up from the plasma at an insulin-independent rate and TAG is taken up into skeletal muscle by being broken down into FFA at an insulin-independent rate. Once inside skeletal muscle, glucose and FFA, as well 


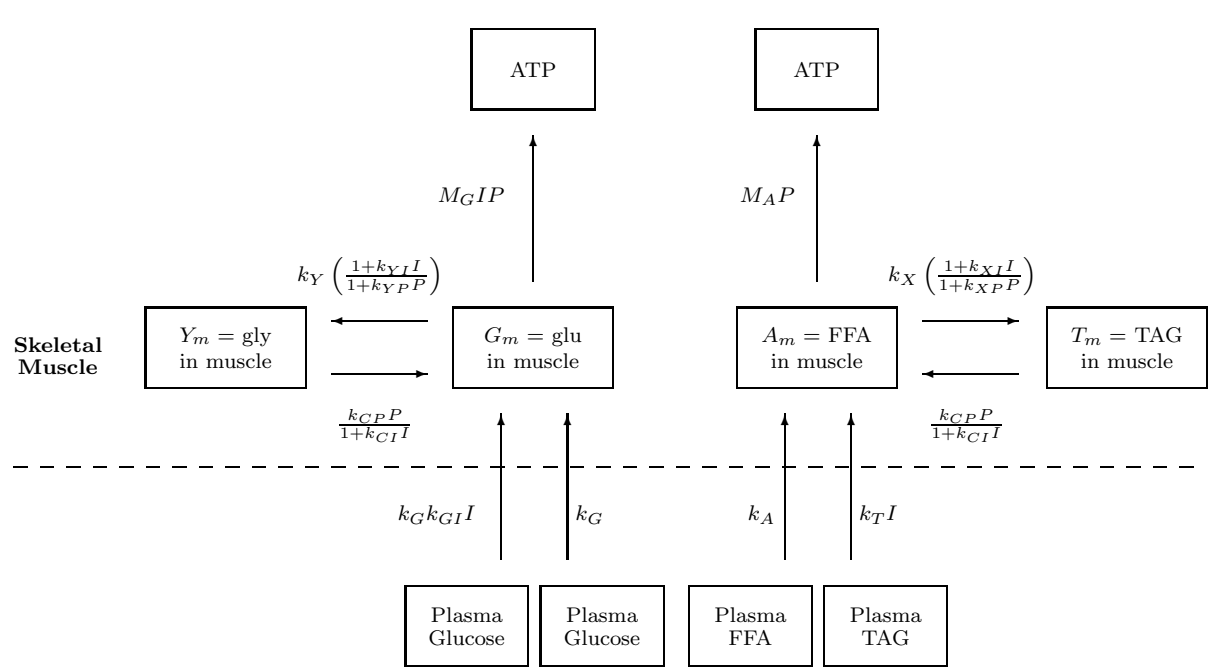

Fig. 3 Diagram showing the input and transportation of glucose, glycogen, FFA and TAG in skeletal muscle with the rate of flux for each pathway shown.

as being oxidised, can be stored as glycogen and TAG respectively, to be released later, when needed. We assume the rate of storage and release of both FFA and glycogen are regulated in the same way, with storage being insulin-stimulated and AMP-inhibited and release being AMP-stimulated and insulin-inhibited. Using these assumptions we arrive at the equations

$$
\begin{aligned}
V_{s} \frac{d G_{m}}{d t} & =k_{G}\left(1+k_{G I} I\right) G_{b}-M_{G} P I G_{m}-k_{Y}\left(\frac{1+k_{Y I} I}{1+k_{Y P} P}\right) G_{m}+\frac{k_{C P} P Y_{m}}{1+k_{C I} I} \\
V_{s} \frac{d Y_{m}}{d t} & =k_{Y}\left(\frac{1+k_{Y I} I}{1+k_{Y P} P}\right) G_{m}-\frac{k_{C P} P Y_{m}}{1+k_{C I} I} \\
V_{s} \frac{d A_{m}}{d t} & =k_{T} T_{b}+k_{A} A_{b}-M_{A} P A_{m}-k_{X}\left(\frac{1+k_{X I} I}{1+k_{X P} P}\right) A_{m}+\frac{k_{D P} P T_{m}}{1+k_{D I} I} \\
V_{s} \frac{d T_{m}}{d t} & =k_{X}\left(\frac{1+k_{X I} I}{1+k_{X P} P}\right) A_{m}-\frac{k_{D P} P T_{m}}{1+k_{D I} I} .
\end{aligned}
$$

Equations (11)-(14) describe the concentration of skeletal muscle glucose, glycogen, FFA and TAG respectively. As with hepatic glycogen, the concentration of skeletal muscle glycogen is actually the concentration of potential glucose stored as glycogen and the concentration of skeletal muscle TAG is actually the concentration of potential FFA stored as TAG. The terms $M_{G} P I G_{m}$ in (11) and $M_{A} P A_{m}$ in (13) describe the use of glucose and fatty acids in the conversion of AMP (and ADP) to ATP, which is then a source of energy for muscle's activity. Protein could also provide a component of the energy required for ATP production, but since our model does not include protein metabolism, we have ignored such effects in this work.

In summary, we have derived twelve first-order coupled differential equations along with 53 dimensional parameters which describe our model. All parameter 


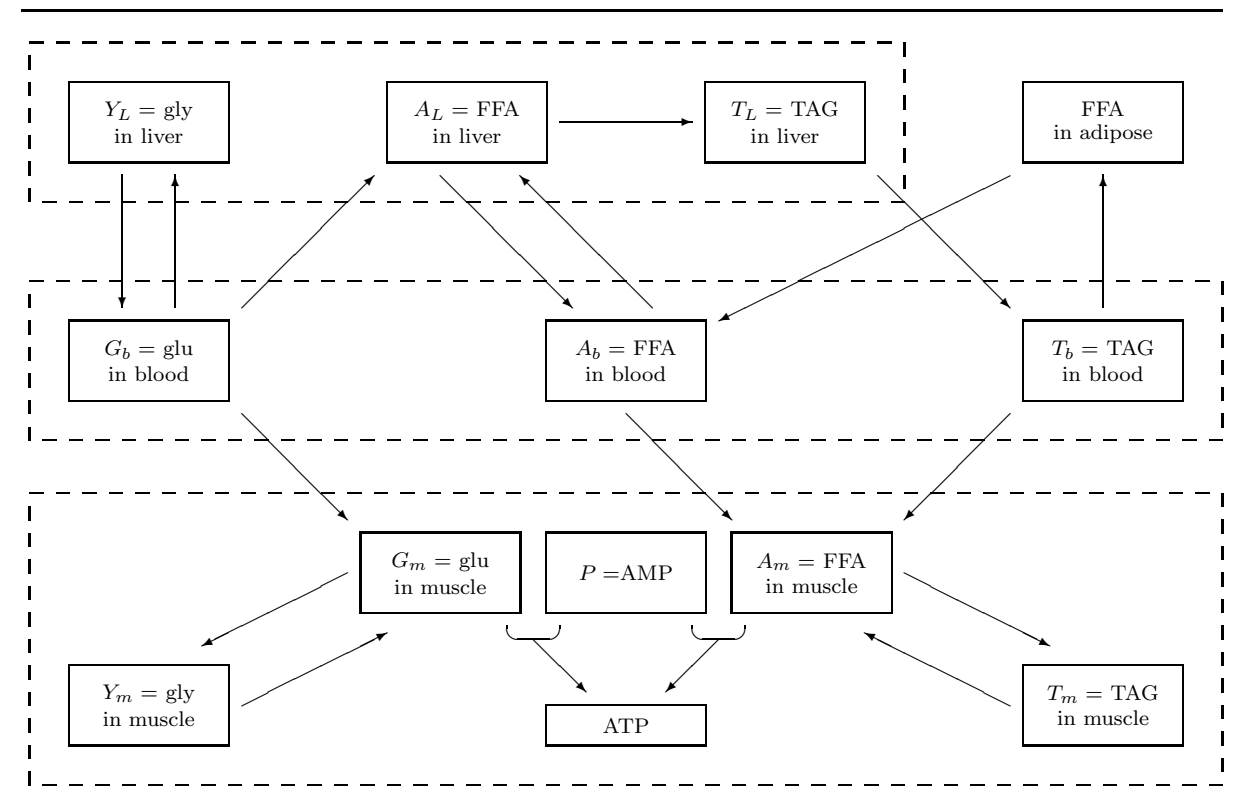

Fig. 4 Diagram of the biochemical reaction network modelled in the rest of this paper. Dashed boxes indicate the different compartments of the model with the liver at the top, the blood plasma in the middle and skeletal muscle at the bottom.

values are given in table 1 along with a brief description of the parameter and a reference to its source where applicable. We summarise the overall model and the interactions between compartments in figure 4.

\section{Steady State}

The steady state we are interested in is the state reached after a 12-14 hour overnight fast. Although hepatic glycogen and hepatic triglyceride will not be at steady state at this time, we assume the stores are sufficiently high that we may approximate the functions $f_{1}, f_{2}$ and $f_{3}$ by 1 , in the case of $f_{2}$ we are also assuming $Y_{L}$ is not close to $Y_{\max }$ (that is, $Y_{0} \ll Y_{\max }-Y_{L}$ ). These assumptions are reasonable for our steady state as $Y_{L}$ will only be near $Y_{0}$ after a period of starvation and will only be near $Y_{\max }$ directly after a meal. Steady state values of variables will be denoted with a superscript "*".

We start by finding the steady states of equations (2) and (3)

$$
\begin{aligned}
G_{b}^{*} & =\frac{\beta_{G}}{\left(1+k_{G L} I^{*^{2}}\right)\left(S_{G}+k_{G}\left(1+k_{G I} I^{*}\right)+k_{L} I^{*}+k_{A L} I^{*}\right)}, \\
T_{b}^{*} & =\frac{\beta_{T}}{\left(1+k_{T L} I^{*}\right)\left(k_{T A}\left(1+k_{A I} I^{*}\right)+k_{T}\right)}
\end{aligned}
$$

Next we solve for the steady states of equations (4) and (8). Solving them explicitly would lead to complicated formulae so we leave the solutions as implicit expressions 
Table 1 List of dimensional parameters together with brief description of each parameter. References are given where a parameter is known from the literature. Parameters without a value given are either eliminated in the nondimensionalisation, are input parameters that we are free to choose. $\dagger$ The value of the parameter $\beta_{T}$ is determined in section 4 using the steady state relations obtained in section 3 .

\begin{tabular}{|c|c|c|c|c|}
\hline Parameter & Description & Value & Units & Reference \\
\hline$M_{G}$ & Glucose oxidation rate & 20000 & $\mathrm{l}^{3} / \min ^{3}$ & \\
\hline$M_{A}$ & FFA oxidation rate & 0.02 & $\mathrm{l}^{2} / \mathrm{min}^{2}$ & \\
\hline$k_{Y}$ & glycogen synthesis rate (basal) & & $1 / \min$ & \\
\hline$k_{Y I}$ & glycogen synthesis rate (insulin stimulated) & 1000000 & $\mathrm{l} / \mathrm{mmol}$ & \\
\hline$k_{Y P}$ & glycogen synthesis rate (AMP inhibited) & 0 & $\mathrm{l} / \mathrm{mmol}$ & \\
\hline$k_{T}$ & muscle triglyceride uptake rate (basal) & $6 \times 10^{-3}$ & $1 / \min$ & [13] \\
\hline$k_{G}$ & muscle glucose uptake rate (basal) & $1.08 \times 10^{-1}$ & $\mathrm{l} / \mathrm{min}$ & {$[18]$} \\
\hline$k_{G I}$ & muscle glucose uptake rate (insulin stimulated) & $1.75 \times 10^{7}$ & $\mathrm{l} / \mathrm{mmol}$ & [18] \\
\hline$k_{C P}$ & glycogenolysis rate (AMP stimulated) & 0.1 & $\mathrm{l}^{2} / \mathrm{min}^{2}$ & \\
\hline$k_{C I}$ & glycogenolysis rate (insulin inhibited) & 0 & $1 / \min$ & {$[17, \mathrm{Ch} .4]$} \\
\hline$S_{G}$ & body glucose consumption rate & & $1 / \min$ & \\
\hline$\mu$ & $\mathrm{AMP}(\mathrm{P})$ creation rate & 1 & $\mathrm{mmol} / \mathrm{min}$ & \\
\hline$\lambda_{I}$ & insulin degradation rate & & $1 / \min$ & \\
\hline$k_{1}$ & insulin production rate & $6.97 \times 10^{-5}$ & $\mathrm{mmol} / \mathrm{min}$ & \\
\hline$k_{2}$ & insulin production rate & $8.36 \times 10^{-5}$ & $\mathrm{mmol} / \mathrm{min}$ & \\
\hline$v$ & insulin production (glucose threshold) & 9 & $\mathrm{mmol} / \mathrm{l}$ & \\
\hline$c$ & insulin production (glucose range) & 4 & $\mathrm{mmol} / \mathrm{l}$ & \\
\hline$k_{I A}$ & insulin production rate & $10^{-6}$ & $1 / \min$ & \\
\hline$k_{A}$ & muscle FFA uptake rate constant & & $1 / \min$ & \\
\hline$\beta_{A}$ & basal FFA production rate & 0.33 & $\mathrm{mmol} / \mathrm{min}$ & [13] \\
\hline$\beta_{T}$ & basal triglyceride production rate & $6.75 \times 10^{-3}$ & $\mathrm{mmol} / \mathrm{min}$ & + \\
\hline$\beta_{G}$ & basal glucose production rate & 0.57 & $\mathrm{mmol} / \mathrm{min}$ & {$[12]$} \\
\hline$\gamma_{a}$ & number of $\mathrm{P}$ molecules used in FFA oxidation & 1 & & \\
\hline$\gamma_{g}$ & number of $\mathrm{P}$ molecules used in glucose oxidation & 1 & & \\
\hline$\lambda_{P}$ & degradation of $\mathrm{P}$ in absence of any other process & & $1 / \min$ & \\
\hline$k_{G L}$ & insulin inhibition rate for glucose from liver & $1.06 \times 10^{14}$ & $\mathrm{l}^{2} / \mathrm{mmol}^{2}$ & {$[18]$} \\
\hline$k_{T L}$ & insulin inhibition rate for TAG from liver & $2.5 \times 10^{6}$ & $\mathrm{l} / \mathrm{mmol}$ & {$[15]$} \\
\hline$k_{A A}$ & insulin inhibition rate for FFA from adipose tissue & $2 \times 10^{14}$ & $\mathrm{l}^{2} / \mathrm{mmol}^{2}$ & [13] \\
\hline$k_{T A}$ & adipose TAG uptake constant (basal) & $1.5 \times 10^{-3}$ & $1 / \min$ & [13] \\
\hline$k_{A I}$ & adipose TAG uptake const. (insulin stimulated) & $5 \times 10^{6}$ & $1 / \mathrm{mmol}$ & [13] \\
\hline$k_{X}$ & muscle TAG synthesis rate constant (basal) & & $1 / \min$ & \\
\hline$k_{X I}$ & muscle TAG synthesis const. (insulin stimulated) & 0 & $\mathrm{l} / \mathrm{mmol}$ & {$[17$, Ch.4] } \\
\hline$k_{X P}$ & muscle TAG synthesis constant (AMP inhibited) & 0 & $\mathrm{l} / \mathrm{mmol}$ & {$[17$, Ch.4] } \\
\hline$k_{D P}$ & muscle TAG usage constant (AMP stimulated) & 0 & $\mathrm{l}^{2} / \mathrm{min}^{2}$ & \\
\hline$k_{D I}$ & muscle TAG usage constant (insulin inhibited) & 0 & $\mathrm{l} / \mathrm{mmol}$ & {$[17$, Ch.4] } \\
\hline$k_{L}$ & liver glucose uptake rate & $6 \times 10^{5}$ & $\mathrm{l}^{2} / \mathrm{min}^{2}$ & {$[12]$} \\
\hline$Y_{\max }$ & maximum potential glucose stored in liver & 310 & $\mathrm{mmol} / \mathrm{l}$ & [21] \\
\hline$V_{b}$ & blood volume & 5 & 1 & \\
\hline$V_{l}$ & liver volume & 1.8 & 1 & [19] \\
\hline$V_{s}$ & skeletal muscle volume & 30 & 1 & {$[20]$} \\
\hline$k_{A L}$ & rate of conversion of glucose to FFA via glycolysis & 2000 & $\mathrm{l}^{2} / \mathrm{mmol} \mathrm{min}$ & \\
\hline$k_{A S}$ & insulin inhibition rate for oxidation of hepatic FFA & $10^{5}$ & $\mathrm{l} / \mathrm{mmol}$ & \\
\hline$k_{B L}$ & rate of uptake of plasma FFA into the liver & 0.034 & $1 / \mathrm{min}$ & \\
\hline$k_{L B}$ & rate of release of hepatic FFA into blood plasma & 0.0059 & $1 / \min$ & \\
\hline$k_{T H}$ & rate of conversion of FFA to TAG in the liver & 33000 & $\mathrm{l}^{2} / \mathrm{mmol} \mathrm{min}$ & \\
\hline$S_{L}$ & rate of oxidation of FFA in the liver & 0.01 & $1 / \min$ & \\
\hline$B_{T}$ & delay from feeding to triglyceride reaching blood & & $\min$ & \\
\hline$B_{G}$ & delay from feeding to glucose reaching blood & & $\min$ & \\
\hline$\theta_{G}$ & amount of carbohydrates in diet & & mmol & \\
\hline$\theta_{T}$ & amount of triglyceride in diet & & mmol & \\
\hline
\end{tabular}


Table 2 List of steady state concentrations for plasma model variables together with reference.

\begin{tabular}{clcl}
\hline Parameter & Description & Value & Reference \\
\hline$G_{b}^{*}$ & Plasma glucose & $5 \mathrm{mmol} / 1$ & {$[17$, Ch. 6] } \\
$T_{b}^{*}$ & Plasma TAG & $1 \mathrm{mmol} / 1$ & {$[17$, Ch. 6] } \\
$A_{b}^{*}$ & Plasma FFA & $0.5 \mathrm{mmol} / 1$ & {$[17$, Ch. 6] } \\
$I^{*}$ & Plasma Insulin & $5 \times 10^{-8} \mathrm{mmol} / 1$ & {$[17$, Ch. 6] } \\
\hline
\end{tabular}

in a form that is useful when we non-dimensionalise

$$
\begin{aligned}
A_{b}^{*} & =\frac{\beta_{A}}{\left(1+k_{A A} I^{* 2}\right)\left(k_{A}+k_{B L}\right)}+\frac{k_{L B} A_{L}^{*}}{k_{A}+k_{B L}}, \\
A_{L}^{*} & =\frac{k_{A L} I^{*} G_{b}^{*}+k_{B L} A_{b}^{*}}{\frac{S_{L}}{1+k_{A S} I^{*}}+k_{L B}+k_{T H} I^{*}} .
\end{aligned}
$$

The steady state solution for insulin is governed by a complicated equation and it is not useful for us to consider the steady state value of this right now as values are commonly available in the literature.

The steady state solutions for equations (11)-(14) are given by

$$
\begin{aligned}
G_{m}^{*}= & \frac{\beta_{G} k_{G}\left(1+k_{G I} I^{*}\right)}{M_{G} P^{*} I^{*}\left(S_{G}+k_{G}\left(1+k_{G I} I^{*}\right)+k_{L} I^{*}+k_{A L} I^{*}\right)}, \\
Y_{m}^{*}= & k_{Y} G_{m}^{*}\left(\frac{1+k_{Y I} I^{*}}{1+k_{Y P} P^{*}}\right)\left(\frac{1+k_{C I} I^{*}}{k_{C P} P^{*}}\right), \\
A_{m}^{*}= & \frac{1}{M_{A} P^{*}}\left(\frac{\beta_{T} k_{T}}{\left(1+k_{T L} I^{*}\right)\left(k_{T A}\left(1+k_{A I} I^{*}\right)+k_{T}\right)}\right) \\
& +\frac{1}{M_{A} P^{*}}\left(\frac{\beta_{A} k_{A}}{\left(1+k_{A A} I^{* 2}\right)\left(k_{A}+k_{B L}\right)}\right)+\frac{1}{M_{A} P^{*}}\left(\frac{k_{A} k_{L B} A_{L}^{*}}{k_{A}+k_{B L}}\right), \\
T_{m}^{*}= & k_{X} A_{m}^{*}\left(\frac{1+k_{X I} I^{*}}{1+k_{X P} P^{*}}\right)\left(\frac{1+k_{D I} I^{*}}{k_{D P} P^{*}}\right) .
\end{aligned}
$$

We can use these equations to find the steady state value for $P$

$$
P^{*}=\frac{\mu-\mu_{c}}{\lambda_{P}}
$$

where $\mu_{c}$ is given by

$$
\begin{aligned}
\mu_{c}= & \frac{\gamma_{A} \beta_{T} k_{T}}{\left(1+k_{T L} I^{*}\right)\left(k_{T A} I^{*}+k_{T}\right)} \\
& +\frac{\gamma_{G} \beta_{G} k_{G}\left(1+k_{G I} I^{*}\right)}{S_{G}+k_{G}\left(1+k_{G I} I^{*}\right)+k_{L} I^{*}+k_{A L} I^{*}} \\
& +\frac{\gamma_{A} \beta_{A} k_{A}}{\left(1+k_{A A} I^{* 2}\right)\left(k_{A}+k_{B L}\right)}+\frac{\gamma_{A} k_{A} k_{L B} A_{L}^{*}}{k_{A}+k_{B L}}
\end{aligned}
$$

and we require $\mu>\mu_{c}$. The steady state values for plasma substrate concentrations, which are widely available in the literature, are given in table 2. 


\section{Parameter Assessment}

Our model has a total of around 50 dimensional parameters, of these: 8 are input parameters which we are free to pick in order to simulate different conditions, 4 are parameters that we can eliminate through nondimensionalisation and the remaining are physiological rate parameters which we must either determine from the literature or, where not known, must choose for ourselves such that the numerical results behave as expected from time courses of experimental data. In this section we describe how we determine some of the model parameters from data available in the literature. We will also make use of some of the results in section 3 by enforcing that some of the equations tend to a steady state, from which we derive a relationship between a group of parameters and the steady state value of a variable. The steady state values of many of our model variables are well documented in the literature so rather than using our estimated parameters to determine the value of the steady state, we will use the well documented steady state values to eliminate the need for us to find all parameters. The standard units of our model are mmol/l for concentrations and time is measured in minutes.

$Y_{\max }$ - The liver is known to be able to store approximately $100 \mathrm{~g}$ of glucose as glycogen [21]. Using an approximate liver volume of 1.8 litres [19] this yields a concentration of $55.5 \mathrm{~g} / \mathrm{l}$. Converting this into the standard units of the model we find $Y_{\max } \approx 310 \mathrm{mmol} / \mathrm{l}$.

$k_{G}, k_{G I}, k_{G L}$ - Meyer et al. [18] studied postprandial glucose homeostasis, analysing the liver, kidneys and skeletal muscle. Healthy subjects were given a 75 gram glucose meal and blood samples were taken over a period of 270 minutes. Fasting plasma glucose and insulin was measured at approximately $5 \mathrm{mmol} / \mathrm{l}$ and $50 \mathrm{pmol} / \mathrm{l}$ respectively and fasting skeletal muscle glucose uptake calculated as approximately $2 \mu \mathrm{mol} / \mathrm{kg} / \mathrm{min}$. Postprandial results showed peak plasma glucose, insulin and skeletal muscle glucose uptake after approximately 90 minutes, with plasma glucose and insulin at approximately $7.5 \mathrm{mmol} / \mathrm{l}$ and $300 \mathrm{pmol} / \mathrm{l} \mathrm{respec}-$ tively and skeletal muscle glucose uptake at approximately $10 \mu \mathrm{mol} / \mathrm{kg} / \mathrm{min}$. This skeletal muscle glucose uptake is given in our model by the term $k_{G}\left(1+k_{G I} I\right) G_{b} / V_{b}$ and as we have data for this rate of uptake, plasma glucose and insulin levels both at the fasted state and at 90 minutes postprandial we can solve simultaneously for the two parameters which gives values of $k_{G} \approx 1.08 \times 10^{-1} \mathrm{l} / \mathrm{min}$ and $k_{G I} \approx 1.75 \times 10^{7} \mathrm{l} / \mathrm{mmol}$. This study also calculated hepatic glucose output and we use this data to calculate $k_{G L}$, the magnitude of hepatic glucose output suppression by insulin. The data calculated hepatic glucose output as $8.34 \mu \mathrm{mol} / \mathrm{kg} / \mathrm{min}$ in the fasted state and as $0.21 \mu \mathrm{mol} / \mathrm{kg} / \mathrm{min}$ in the postprandial state. As stated before we also know the concentrations of insulin at these times and we can pick our parameter such that the measured increase in insulin levels results in a suppression of hepatic glucose output in agreement with the data. This yields an estimate of $k_{G L} \approx 1.06 \times 10^{14} \mathrm{l}^{2} / \mathrm{pmol}^{2}$.

$\beta_{G}, k_{L}$ - Taylor et al. [12] carried out a study into hepatic glycogen storage in healthy subjects. Three different studies where done order to measure different rates and concentrations. We will be interested in the data from study A as this had the most simple protocol. Subjects were fed a liquid meal of approximately $550 \mathrm{kcal}$ carbohydrates and $150 \mathrm{kcal}$ fat following an overnight fast. Hepatic glycogen levels were then measured over the next 10 hours using magnetic resonance spectroscopy. The results showed a steady rise in hepatic glycogen concentration during the 
insulin stimulated postprandial period to near the liver storage limit, followed by a steady decline in hepatic glycogen over the remainder of the 10 hours. We pick our parameters such that subject to the same simulated meal our results show a qualitative agreement with this data, in particular that hepatic glycogen rises following the meal until almost full followed by a steady decline returning to its initial value after a $12-14$ hour fast. This gives estimates for the parameters of $\beta_{G} \approx 0.57 \mathrm{mmol} / \mathrm{min}$ and $k_{L} \approx 6 \times 10^{5} \mathrm{l}^{2} / \mathrm{mmol} / \mathrm{min}$.

$k_{T}, k_{T A}, k_{A I}, \beta_{A}, k_{A A}$ - Bickerton et al. [13] carried out a study looking into fatty acid metabolism in healthy and insulin-resistant men. The study was carried out after an overnight fast followed by a test meal of $40 \mathrm{~g}$ fat and $40 \mathrm{~g}$ carbohydrate. Blood samples were taken for 6 hours after ingestion of the meal (initially every 30 minutes and then every 60 minutes) and results for plasma concentrations recorded as well as a calculation of the rate of clearance of TAG from the plasma into adipose tissue and skeletal muscle. Comparing the results for skeletal muscle TAG clearance to the plasma TAG concentration confirmed that a linear relationship between skeletal muscle TAG uptake and plasma TAG concentration was a good model. After converting the units of the study ( $\mathrm{ml} / \mathrm{min} / 100 \mathrm{~g}$ tissue) into the units of our model we are able to derive a value for $k_{T}$ by finding the value of $k_{T} / V_{b}$ that maps the results for plasma TAG concentration to the results of skeletal muscle TAG clearance. This gives a value for $k_{T}$ of $6 \times 10^{-2} \mathrm{l} / \mathrm{min}$. However the results for adipose tissue TAG clearance show a behaviour that our current model is unable to simulate and show an immediate spike in adipose TAG uptake which then returns towards steady state value before raising again later, coinciding with the peak in plasma TAG levels. The data also shows a third rise in adipose TAG uptake towards the end of the data at approximately 6 hours after the test meal when TAG and insulin levels have returned to steady state. We nevertheless find values for $k_{T A}$ and $k_{A I}$ that produces as good a fit with the experimental data as possible, in particular our model predicts rates of uptake within the same range of values as the data. We use the same approach as with skeletal muscle uptake and take the data for plasma TAG and also insulin and pick values for the parameters which make the quantity $k_{T A}\left(1+k_{A I} I\right) T_{b} / V_{b}$ in good agreement with the experimental data. Using $k_{T A}=1.5 \times 10^{-2} \mathrm{l} / \mathrm{min}$ and $k_{A I}=5 \times 10^{6} \mathrm{l} / \mathrm{mmol}$ yields results in the same range as the experimental data however this does not show good qualitative agreement, in particular the third spike in adipose tissue TAG uptake. Also of use to us from this study is the results for adipose tissue NEFA (FFA) output. Fasting adipose NEFA output was measured at approximately $1200 \mathrm{nmol} /$ minute $/ 100 \mathrm{~g}$ adipose tissue, at which time plasma insulin was measured at approximately $50 \mathrm{pmol} / \mathrm{l}$. At approximately 120 minutes after the meal was ingested adipose NEFA output reached its minimum of approximately $200 \mathrm{nmol} / \mathrm{min} / 100 \mathrm{~g}$ adipose tissue, this coincides with the maximum plasma insulin concentration measured, approximately $200 \mathrm{pmol} / \mathrm{l}$. Adipose tissue NEFA output is given in our model by the term $\beta_{A} /\left(V_{b}\left(1+k_{A A} I^{2}\right)\right)$ and as we have data for this rate of release and insulin levels both at the fasted state and 120 minutes postprandial we can solve simultaneously for the two parameters which gives values of $\beta_{A} \approx 0.33 \mathrm{mmol} / \mathrm{min}$ and $k_{A A} \approx 2 \times 10^{14} \mathrm{l}^{2} / \mathrm{pmol}^{2}$.

$\beta_{T}, k_{T L}$ - Here we make use of the steady state relation given in equation (15), as we have estimates for the parameters $k_{T}, k_{T A}$ and $k_{A I}$ and we know the steady 
state value of plasma TAG, $T_{b}^{*}$, we can derive that

$$
\begin{aligned}
\frac{\beta_{T}}{1+k_{T L} I^{*}} & =\left(k_{T}+k_{T A}\left(1+k_{A I} I^{*}\right)\right) T_{b}^{*}, \\
& =6 \times 10^{-3} \mathrm{mmol} / \mathrm{min} .
\end{aligned}
$$

Now, as we have two parameters to find and only one equation we require a little more information. Adiels et al. [15] carried out a study into the suppression of hepatic VLDL1 secretion by insulin, and of particular use to us VLDL-TAG is measured. This secretion of VLDL1 relates in our model to the release of TAG from the liver; although the other types of lipoprotein are involved in TAG transport, VLDL1 is responsible for the majority of the flux through the insulin inhibited hepatic secretion pathway. Their study compared results for subjects with type- 2 diabetes to control subjects under conditions of an euglycemic hyperinsulinemic clamp following an overnight fast. Plasma insulin for the control subjects was measured at approximately $50 \mathrm{pmol} / \mathrm{l}$ at the start of the clamp and $500 \mathrm{pmol} / \mathrm{l}$ once a steady state was achieved. VLDL1-TAG secretion rate for the control subjects was measured at approximately $250 \mathrm{mg} / \mathrm{kg} /$ day at the start of the clamp and 125 $\mathrm{mg} / \mathrm{kg} /$ day once a steady state was reached. We can simplify this data by saying that a 10 fold increase in plasma insulin, from its steady state value, causes hepatic TAG output to drop to $50 \%$ of its steady state value. Combining this observation with equation (25) gives a value for $\beta_{T}$ of $6.75 \times 10^{-2} \mathrm{mmol} / \mathrm{min}$ and a value for $k_{T L}$ of $2.5 \times 10^{6} \mathrm{l} / \mathrm{mmol}$.

\section{Numerical Results}

In this section we solve our model numerically subject to two different simulated meals. Hence we must pick our input functions, $F_{G}(t)$ and $F_{T}(t)$, appropriately and it will be useful for us to consider them in terms of energy (kcal).

The total amount of glucose entering the system from the diet is given by

$$
G_{i n}=\frac{1}{V_{b}} \int_{0}^{\infty} F_{G}(t) d t .
$$

However as we are solving the nondimensional system we need to transform the integral to nondimensional variables which gives

$$
G_{i n}=G_{b}^{*} \int_{0}^{\infty} \widehat{F}_{G}(\widehat{t}) d \widehat{t} .
$$

Now in order to convert this result from the units of our model, namely mmol/l (blood), into energy we need to know the total volume of blood, the mass in grams of one $\mu \mathrm{mol}$ of glucose and the amount of kcal per gram of glucose, which we will denote by $V_{b}, R_{g}$ and $C_{g}$ respectively. This gives the following expression for total energy from glucose entering the system from the diet

$$
G_{c a l}=C_{g} R_{g} V_{b} G_{b}^{*} \int_{0}^{\infty} \widehat{F}_{G}(\widehat{t}) d \widehat{t}
$$

Using a similar derivation for TAG entering the system from the diet gives

$$
T_{c a l}=\frac{\beta_{T} C_{t} R_{t} V_{b} G_{b}^{*}}{\beta_{G}} \int_{0}^{\infty} \widehat{F}_{T}(\widehat{t}) d \widehat{t},
$$


Table 3 List of parameters used to determine calorific content of simulated meal.

\begin{tabular}{clcl}
\hline Parameter & Description & Value & Reference \\
\hline$V_{b}$ & Total volume of blood & $5 \mathrm{l}$ & \\
$R_{g}$ & Mass of one mmol glucose & $0.18 \mathrm{~g}$ & {$[17$, Ch. 6] } \\
$C_{g}$ & Kcal per gram glucose & $4 \mathrm{kcal}$ & {$[17$, Ch. 6] } \\
$R_{t}$ & Mass of one mmol TAG & $0.85 \mathrm{~g}$ & {$[17$, Ch. 6] } \\
$C_{t}$ & Kcal per gram TAG & $9 \mathrm{kcal}$ & {$[17$, Ch. 6] } \\
\hline
\end{tabular}
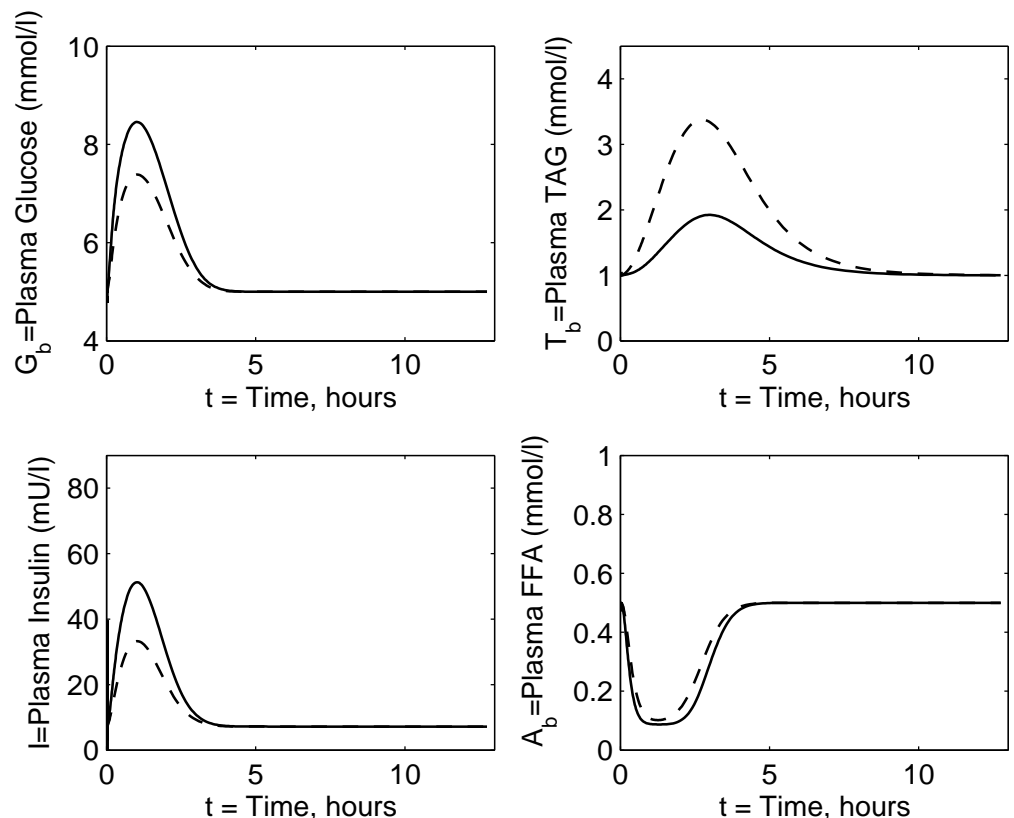

Fig. 5 Post-prandial kinetics for plasma glucose (top left), plasma insulin (bottom left), plasma FFA (bottom right) and plasma TAG (top right) for a balanced meal (solid line) and a high fat meal (dashed line). Time is plotted in hours.

where $R_{t}$ and $C_{t}$ denote the mass in grams of one mmol of TAG and the calories per gram of TAG respectively. These parameters and their values are listed in table 3 .

Using the above results we simulate a "healthy" balanced meal and a high fat meal and solve the model numerically in matlab. The initial conditions we take as being the steady state after a $12-14$ hour overnight fast. For the balanced meal we choose the input functions to simulate a meal of $550 \mathrm{kcal}$ carbohydrates (glucose) and $150 \mathrm{kcal}$ of fat (TAG). For the high fat meal we simulate $300 \mathrm{kcal}$ of carbohydrates and $300 \mathrm{kcal}$ of fat. Before solving the governing equations numerically, the system is nondimensionalised, the details of which are given in Appendix B.

The results for the plasma concentrations are shown in figure 5 and show good qualitative agreement with experimental data, both in post prandial behaviour and in the timescales to return to steady state [17, Ch. 6]. Only concentrations are plotted here: since most fluxes are of the form constant times a concentration, the evolution of fluxes simply follow the corresponding concentration. In Appendix C, we plot more complex combinations of concentrations which describe the other 

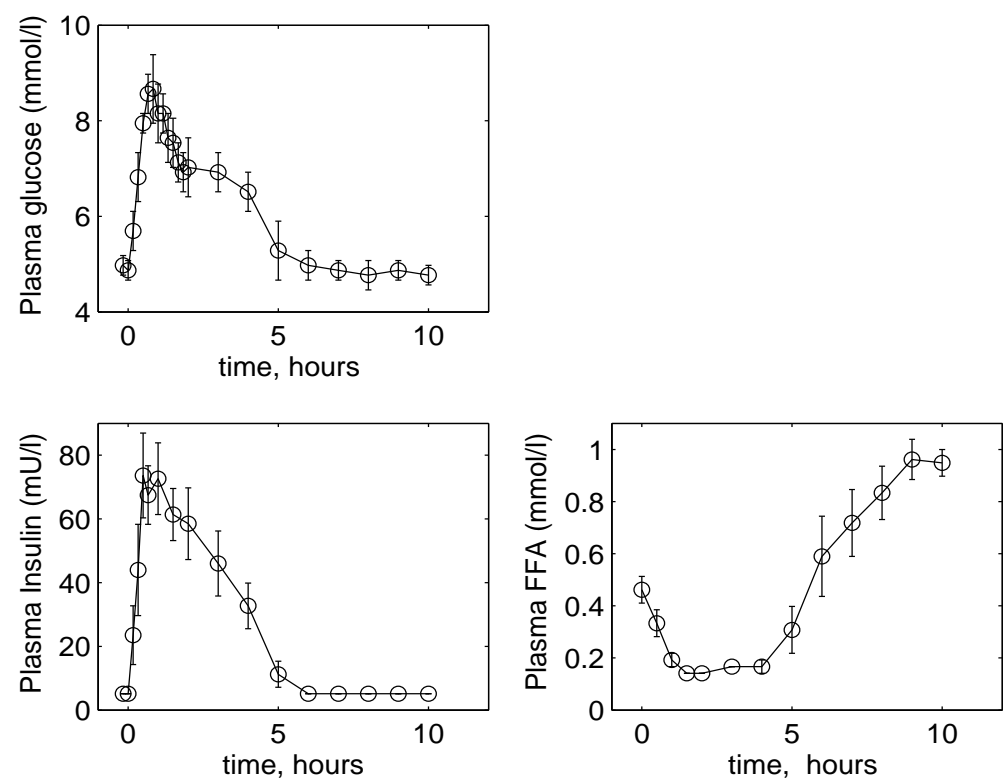

Fig. 6 Experimental post-prandial kinetic data for plasma glucose (top left), plasma insulin (lower left), plasma FFA (lower right), all plotted against time (hours). Taken from Taylor et al. [12].

fluxes in the system. The quantitative agreement with experimental data for these results is fairly good, as can be seen by comparing Figure 5 with the experimental data of Taylor et al [12] plotted in Figure 6. Comparing the two meals we see that plasma insulin and glucose concentrations do not reach as high in the high fat meal as in the balanced meal, due to less glucose entering the system which also directly affects the rate of insulin secretion. Plasma FFA has less of a decline in the high fat meal due to the lower levels of insulin producing a smaller reduction in the adipose tissue FFA output and plasma TAG concentration is higher in the high fat meal.

The results for hepatic concentrations are shown in figure 7. Hepatic glycogen shows good qualitative and quantitative agreement with experimental data [12]. Following ingestion of the meal glucose is taken up from the plasma to be stored in the liver and within a few hours the liver is almost full to its maximum. Then follows several hours of the store being slowly depleted as the glycogen is released back into the plasma as glucose. Although we have no data to compare the results for hepatic TAG with, these results seem to agree with our intuition, that following a meal the liver TAG concentration would rise and then slowly deplete as the TAG is released back into the plasma. The magnitude by which this happens is also in agreement with the fact that liver fat content is not heavily affected by a single meal but rather by a consistent unhealthy diet, which would result in a significant build up of liver fat. Similarly, for the results for hepatic FFA, we have no data to compare this to, however, the simulated results agree with our intuition. Following ingestion of the meal, plasma FFA concentration decreases, this in turn causes the hepatic FFA concentration to also decrease. Of particular interest is the simulated 

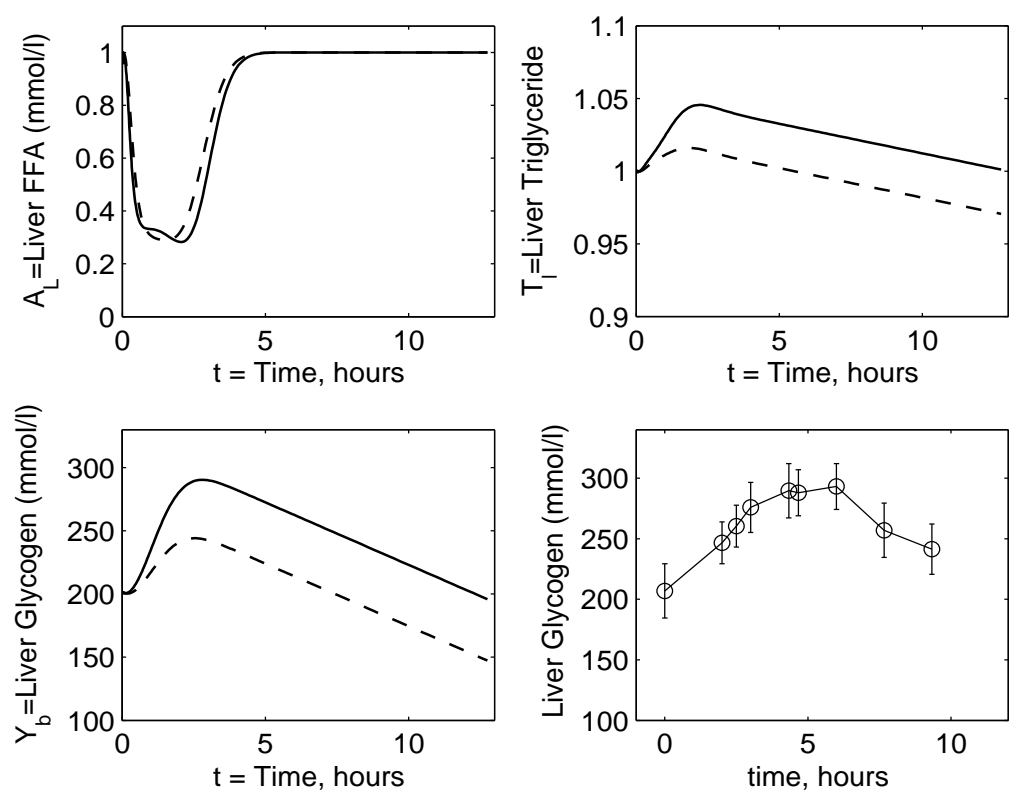

Fig. 7 Post-prandial kinetics for hepatic FFA (top left) hepatic TAG (top right) and hepatic glycogen (lower left), for a balanced meal (solid line) and a high fat meal (dashed line). Experimental data for hepatic glycogen from Taylor et al. [12] is shown in the lower right plot. In all plots, time is measured in hours.

"two stage" reduction in hepatic FFA which is due to the uptake and conversion of plasma glucose into FFA in the liver partially counteracting the decrease in concentration caused by the lower plasma FFA levels. Comparing the results for the two meals we see that in the high fat meal, the shortage of glucose entering the system means that hepatic glycogen does not reach its maximum after the meal and over the course of the 12-14 hour simulation the liver has an overall net loss of glycogen. The effects of the lower levels of plasma glucose and insulin can also be seen in the results for hepatic FFA and TAG, with not enough glucose being taken up into the liver and converted to FFA in order to see the two stage decline in hepatic FFA. Interestingly we see that in the high fat meal the liver gains less TAG and over the 12-14 hours actually loses TAG, this is due to the conversion of hepatic FFA into TAG being an insulin driven pathway.

The results for skeletal muscle concentrations are shown in figure 8. We currently have no data to compare the results for all substrates in skeletal muscle with. The results for skeletal muscle glucose and glycogen rise after ingestion of the meal which would seem intuitive due to the increase in plasma glucose and insulin. The results for skeletal muscle FFA and TAG show that, despite an elevated level of plasma TAG, the uptake of TAG from the plasma is not enough to compensate for the reduced uptake of FFA from the plasma. Whether or not this is what happens in vivo we are unable to say at this time as we have no data to compare it to. In the case of the high fat meal we do however see that after the initial decline in muscle FFA and TAG that we get a slight increase above the steady state value, this is due to the timescale for appearance in, and subsequently 

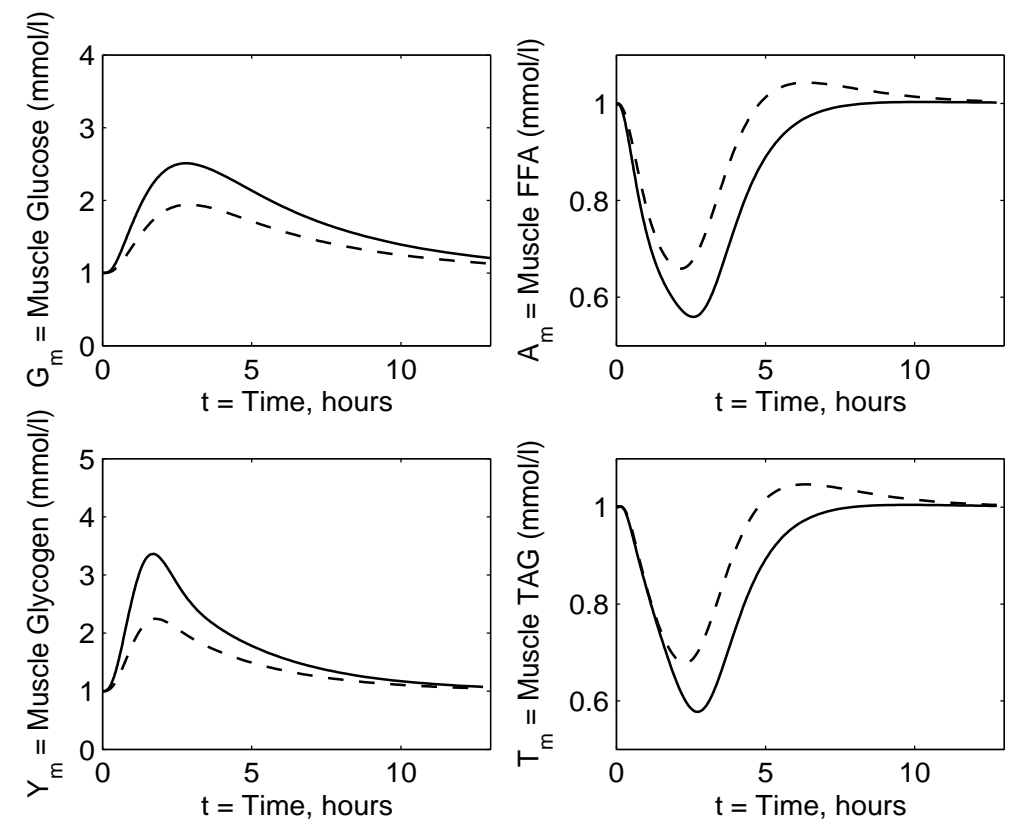

Fig. 8 Post-prandial kinetics for muscle glucose (top left), muscle glycogen (bottom left), muscle FFA (top right) and muscle TAG (bottom right) for a balanced meal (solid line) and a high fat meal (dashed line), plotted against time (in hours).

removal from, the plasma of TAG being on a longer timescale than glucose and insulin. There are data for metabolic flexibility in skeletal muscle for which we can compare our model results to. There are well documented data for respiratory quotient in skeletal muscle $(\Delta R Q)$, these in effect measure how much of the total oxidation in the muscle is due to glucose oxidation. We can define a term from our model which is analogous to a respiratory quotient which we define by

$$
S_{W}=\frac{M_{G} P I G_{m}}{M_{A} P A_{m}+M_{G} P I G_{m}}=\frac{M_{G} I G_{m}}{M_{A} A_{m}+M_{G} I G_{m}} .
$$

The results for this "metabolic flexibility" are shown in figure 9. These results show very good agreement with experimental data [8], with approximately one third of oxidation being due to glucose oxidation in the fasted state and almost $90 \%$ of oxidation being due to glucose in the post prandial period in the case of the balanced meal. As we would expect, in the case of the high fat meal we see less of a switching in fuel source, however in the post prandial state glucose is still the predominant fuel source.

One area that we simplify in our model which is of interest to us is the adipose tissue. We do not take account of any concentrations inside adipose tissue nor do we account for varying volumes of adipose tissue. We can however take some data from our simulations to get an idea of the effect of the different meals on adipose tissue by looking specifically at adipose tissue TAG uptake. We will produce the results for adipose tissue TAG uptake in dimensional form by plotting the following expression

$$
k_{T A}\left(1+k_{A I} I^{*} I\right) T_{b}^{*} T_{b} .
$$



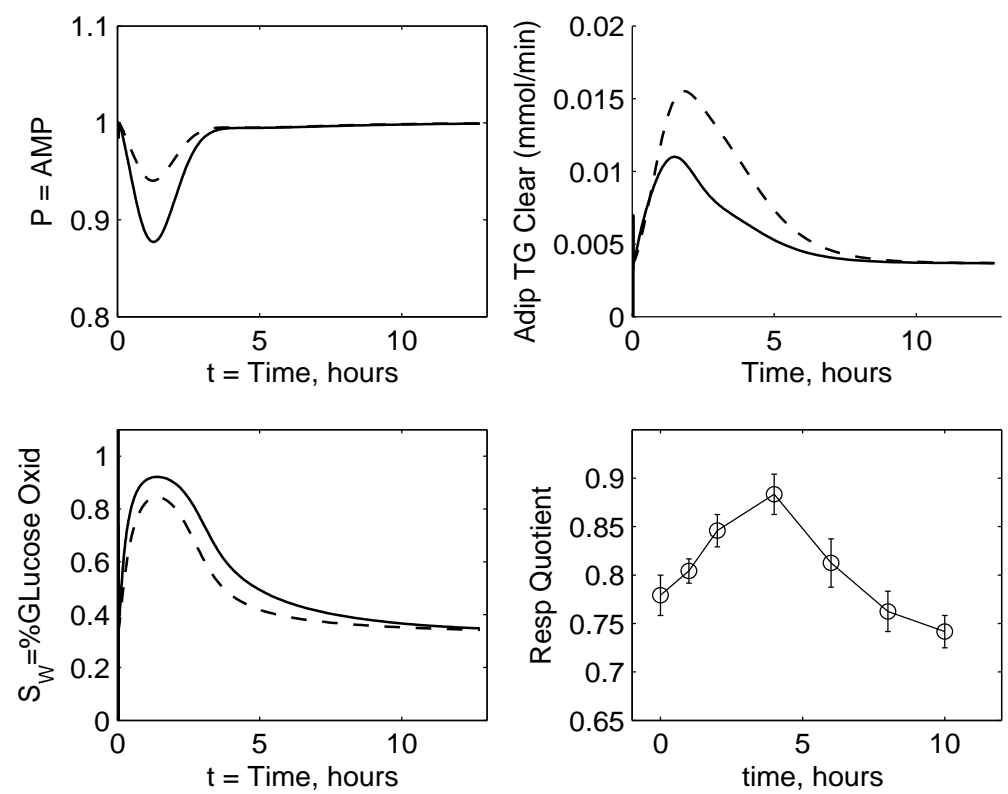

Fig. 9 Post prandial $P$ (AMP) (top left) and post prandial adipose tissue TAG uptake (top right) for a balanced meal (solid line) and a high fat meal (dashed line). Post-prandial metabolic flexibility (lower left), assessed by the percentage of total oxidation due to glucose oxidation, and experimental data on respiratory quotient, taken from Taylor et al. [12]. In all panels, time is in hours. $P$, being a proxy for AMP, is left dimensionless.

The results for adipose tissue TAG clearance are shown in figure 9 and we can see that following a meal some of the excess fat in the blood plasma is taken up into the adipose tissue for storage. This rate is given by the product $k_{T A} T_{b}\left(1+k_{A I} I\right)$, and the form can be deduced from the graphs in Figure 5 . In the case of the high fat meal we can see there is a significant increase in the amount of fat taken up into adipose tissue, despite the lower levels of plasma insulin and an overall lower total calorific intake, due to the increased plasma TAG concentrations. In contrast, the rate of triglyceride clearance in muscle is given by $k_{D P} P T_{m} /\left(1+k_{D I} I\right)$, whose constituent concentrations can be seen in Figures $5(I), 8\left(T_{m}\right)$ and $9(P)$, from these graphs we note that in the range $0<t<10$ TAG clearance is higher during high fat meal. Note, lower insulin levels during a high fat meal gives rise to the counterbalancing possibility that high fat meals cause greater lipolysis of adipose tissue.

\section{Conclusions}

We have developed a compartmental model of human metabolism including substrate transport and utilisation in the plasma, the liver and skeletal muscle. Our simulated results have been shown to be in good agreement with experimental data, in particular the results for plasma concentrations and hepatic glycogen. The fact that these are the substrates for which we can find many of the rate parameters from the literature is encouraging. In the case of some of the rates, 
in particular those related to glucose and insulin, there is an abundance of data available from the literature which implies that our modelling assumptions are correct. We have stated that for some other results, although we currently have no data to compare with, our model predictions agree with our intuition. In the case of hepatic FFA and TAG we must be careful with how we assign these parameters and what we define as intuitively correct, as the transport to and from, and the conversion in, the liver are heavily dependent on what happens in the plasma. In contrast to this, although we currently have little data on what happens in skeletal muscle, this is not so important since the equations for plasma and hepatic concentrations have no dependence on the skeletal muscle concentrations. In effect, we really have two subsystems, the plasma-liver subsystem and the skeletal muscle subsystem, which is driven by the plasma-liver subsystem.

In recent years there has been much mathematical modelling of human metabolism, however, most of this work focuses on smaller scale, localised modelling, describing in detail certain systems or pathways involved with metabolism and in some cases focusing on kinetics on the cellular level. In this paper we have taken a larger scale approach to modelling metabolism and derived equations to link together most of the different pathways involved with metabolism of carbohydrates and fats by taking a less detailed approach but still keeping account of the important stages in metabolism. The model does not model the increase or decrease in adipose tissue over large times, it is not designed to describe the long term starvation. Rather, our aim is to provide a more detailed understanding of metabolic flexibility.

We developed a system of 12 first-order coupled differential equations which describe human metabolism across the whole body. With important tissues such as the liver and skeletal muscle modelled in detail and the rest of the body simplified into single terms. We found steady state solutions to the system and used these solutions to nondimensionalise the system which reduces the number of parameters which influence the models kinetic behaviour. We found numerical results for the model based on ingestion of a "balanced" meal and highlighted areas where our model results are in agreement with experimental data as well as areas where we are unsure of the physiological behaviour due to a lack of data.

Future work on this model will include calibrating the model to simulate insulin-resistance in order to produce a variety of results for different levels of insulin resistance, different combinations of tissues insulin resistant. We shall also look at different combinations of meals and meal compositions. In addition, we plan to expand the model to give a more complete model of human metabolism by including a more detailed study AMP oxidation, lactate transport between skeletal muscle and the liver, gluconeogenesis in the liver, including other regulatory hormones (such as glucagon) and modelling metabolism in adipose tissue [48].

Acknowledgements TP acknowledges support from BBSRC and Unilever Corporate Research. The authors are grateful to the referees for their helpful comments on earlier versions of the manuscript. 


\section{A Properties of solutions of the governing equations}

\section{A.1 Uniqueness of Steady State}

In this appendix we will prove the uniqueness of our steady states. We begin by writing down which variables each steady state is a function of (except $I^{*}$ ),

$$
\begin{aligned}
G_{b}^{*} & =\frac{\beta_{G}}{\left(1+k_{G L} I^{* 2}\right)\left(S_{G}+k_{G}\left(1+k_{G I} I^{*}\right)+k_{L} I^{*}+k_{A L} I^{*}\right)}=f_{1}\left(I^{*}\right), \\
T_{b}^{*} & =\frac{\beta_{T}}{\left(1+k_{T L} I^{*}\right)\left(k_{T A}\left(1+k_{A I} I^{*}\right)+k_{T}\right)}=f_{2}\left(I^{*}\right), \\
A_{l}^{*} & =\frac{k_{A L} I^{*} G_{b}^{*}+k_{B L} A_{b}^{*}}{\frac{S_{L}}{1+k_{A S} I^{*}}+k_{L B}+k_{T H} I^{*}}=f_{3}\left(I^{*}, G_{b}^{*}, A_{b}^{*}\right), \\
A_{b}^{*} & =\frac{\beta_{A}}{\left(1+k_{A A} I^{* 2}\right)\left(k_{A}+k_{B L}\right)}+\frac{k_{L B} A_{L}^{*}}{k_{A}+k_{B L}}=f_{4}\left(I^{*}, A_{l}^{*}\right), \\
G_{m}^{*} & =\frac{\beta_{G} k_{G}\left(1+k_{G I} I^{*}\right)}{M_{G} P^{*} I^{*}\left(S_{G}+k_{G}\left(1+k_{G I} I^{*}\right)+k_{L} I^{*}+k_{A L} I^{*}\right)}=f_{5}\left(I^{*}, P^{*}\right), \\
A_{m}^{*} & =\frac{1}{M_{A} P^{*}}\left(\frac{\beta_{T} k_{T}}{\left(1+k_{T L} I^{*}\right)\left(k_{T A}\left(1+k_{A I} I^{*}\right)+k_{T}\right)}\right)=f_{6}\left(I^{*}, P^{*}\right), \\
Y_{m}^{*} & =k_{Y} G_{m}^{*}\left(\frac{1+k_{Y I} I^{*}}{1+k_{Y P} P^{*}}\right)\left(\frac{1+k_{C I} I^{*}}{k_{C P} P^{*}}\right)=f_{7}\left(I^{*}, P^{*}, G_{m}^{*}\right), \\
T_{m}^{*} & =k_{X} A_{m}^{*}\left(\frac{1+k_{X I} I^{*}}{1+k_{X P} P^{*}}\right)\left(\frac{1+k_{D I} I^{*}}{k_{D P} P^{*}}\right)=f_{8}\left(I^{*}, P^{*}, A_{m}^{*}\right), \\
P^{*} & =\frac{\mu-\mu_{c}}{\lambda_{P}}=f_{9}\left(I^{*}\right) .
\end{aligned}
$$

The expressions for $A_{b}^{=} f_{4}$ and $A_{l}^{*}=f_{3}$ form a linear system for $A_{b}^{*}$ and $A_{l}^{*}$ which has a unique solution for each quantity in terms of $I^{*}$ and $G_{b}^{*}$, and hence the solutions can be written explicitly in terms of $I^{*}$ using $G_{b}^{*}=f_{1}\left(I^{*}\right)$. Now we substitute the expression for $P^{*}=f_{9}$ into the equations for $G_{m}^{*}=f_{5}, A_{m}^{*}=f_{6}, Y_{m}^{*}=f_{7}$, etc. to obtain a simpler system given by

$$
\begin{gathered}
G_{b}^{*}=f_{1}\left(I^{*}\right), \quad T_{b}^{*}=f_{2}\left(I^{*}\right), \quad A_{l}^{*}=\widetilde{f}_{3}\left(I^{*}\right), \quad A_{b}^{*}=\widetilde{f}_{4}\left(I^{*}\right), \quad G_{m}^{*}=\widetilde{f}_{5}\left(I^{*}\right), \\
A_{m}^{*}=\widetilde{f}_{6}\left(I^{*}\right), \quad Y_{m}^{*}=\widetilde{f}_{7}\left(I^{*}, G_{m}^{*}\right), \quad T_{m}^{*}=\widetilde{f}_{8}\left(I^{*}, A_{m}^{*}\right), \quad P^{*}=f_{9}\left(I^{*}\right) .
\end{gathered}
$$

Thus all quantities can now be written as a function of $I^{*}$, only $T_{m}^{*}$ still depend on other quantities, and these can be eliminated using other expressions in (33), for example, $T_{m}^{*}=$ $\tilde{f}_{8}\left(I^{*}, \tilde{f}_{6}\left(I^{*}\right)\right)$.

Considering the steady-state equation for insulin, we write down an expression for $I^{*}$,

$$
I^{*}=\frac{k_{1}+k_{2} \operatorname{erf}\left(\left(G_{b}-v\right) / c\right)+k_{I A} A_{b}^{*}}{\lambda_{I}} .
$$

We now examine the steady state values for $G_{b}^{*}$ and $A_{b}^{*}$ given in table 2 and note that $G_{b}$ terms contribute more significantly to the steady state than the $A_{b}^{*}$ terms. Hence we temporarily neglect the $A_{b}^{*}$ term. Under this assumption we substitute in the expression for $G_{b}^{*}$ to find

$$
\lambda_{I} I^{*}=k_{1}+k_{2} \operatorname{erf}\left(\frac{f_{1}\left(I^{*}\right)-v}{c}\right) .
$$

The left hand side of equation 35 is an increasing function of $I^{*}$ for $I^{*}>0$, satisfying RHS $=0$ when $I^{*}=0$, and the right hand side is a decreasing function of $I^{*}$ for $I^{*}>0$, with RHS $>0$ at $I^{*}=0$; therefore there is a unique solution for $I^{*}$. 
Given that $I^{*}$ has a unique solution, we can now follow the chain of reasoning

$I^{*}$ has unique steady-state $\Rightarrow G_{b}^{*}, T_{b}^{*}, P^{*}$ have unique steady-states,

$I^{*}$ and $P^{*}$ have unique steady-states $\Rightarrow G_{m}^{*}$ and $A_{m}^{*}$ have unique steady-states,

$I^{*}$ and $G_{b}^{*}$ have unique steady-states $\Rightarrow A_{l} *$ and $A_{b}^{*}$ have unique steady-state,

$I^{*}, P^{*}$ and $G_{m}^{*}$ have unique steady-states $\Rightarrow Y_{m}^{*}$ has unique steady-state ,

$I^{*}, P^{*}$ and $A_{m}^{*}$ have unique steady-states $\Rightarrow T_{m}^{*}$ has unique.

Hence the system as whole has a unique steady-state solution.

Reinstating the $A_{b}^{*}$ term theoretically could make the RHS of (35) nonmonotone, and so there could be multiple steady-states, however, for the physically realistic parameter values of interest to us, this does not occur.

\section{A.2 Positivity of solutions}

Since all of the governing equations (1)-(4), (7)-(14) have the form $\mathrm{d} X / \mathrm{d} t=A-B X$ with $A>0$, we can be sure that if $X=0$ ever occurs, then $X$ would increase, and so, provided we start with positive initial data, the concentrations will remain positive for all time.

Since the nonlinearities in the model are all analytic and have at most linear growth, the standard theory of ordinary differential equations implies uniqueness for the initial value problem.

\section{B Non-dimensionalisation}

Before we attempt to solve the system numerically, we nondimensionalise it so that we may reduce the number of parameters in the model. This process also allows for simpler numerical simulations. We rescale each variable by its steady state value, except for $Y_{L}$ which we rescale by $Y_{\max }$, and $T_{L}$ which we rescale by a typical healthy liver fat concentration, denoted by $T_{L}^{H}$. This means that we are now concerned with the following non-dimensional variables

$$
\begin{gathered}
\widehat{Y}_{L}=\frac{Y_{L}}{Y_{\max }}, \quad \widehat{A}_{L}=\frac{A_{L}}{A_{L}^{*}}, \quad \widehat{T}_{L}=\frac{T_{L}}{T_{L}^{H}}, \quad \widehat{G}_{b}=\frac{G_{b}}{G_{b}^{*}}, \\
\widehat{A}_{b}=\frac{A_{b}}{A_{b}^{*}}, \quad \widehat{T}_{b}=\frac{T_{b}}{T_{b}^{*}}, \quad \widehat{I}=\frac{I}{I^{*}}, \quad \widehat{G}_{m}=\frac{G_{m}}{G_{m}^{*}}, \quad \widehat{Y}_{m}=\frac{Y_{m}}{Y_{m}^{*}}, \\
\widehat{Y}_{m}=\frac{Y_{m}}{Y_{m}^{*}}, \quad \widehat{A}_{m}=\frac{A_{m}}{A_{m}^{*}}, \quad \widehat{T}_{m}=\frac{T_{m}}{T_{m}^{*}}, \quad \widehat{P}=\frac{P}{P_{b}^{*}}, \quad t=\frac{V_{b} G_{B}^{*}}{\beta_{G}} \widehat{t} .
\end{gathered}
$$

The forcing functions are nondimensionalised by $F_{G}(t)=\beta_{G} \widehat{F}_{G}(\widehat{t})$ and $F_{T}(t)=\beta_{T} \widehat{F}_{T}(\widehat{t})$, where the nondimensional forcing functions are given by

$$
\begin{aligned}
& \widehat{F}_{G}(\widehat{t})=\frac{\widehat{k}_{F G} \widehat{t}}{\widehat{\tau}_{G}} e^{-\widehat{t}^{2} / 2 \widehat{\tau}_{G}^{2}}, \quad \widehat{k}_{F G}=\frac{\theta_{G}}{B_{G} \beta_{G}}, \quad \widehat{\tau}_{G}=\frac{B_{G} \beta_{G}}{V_{b} G_{B}^{*}}, \\
& \widehat{F}_{T}(\widehat{t})=\frac{\widehat{k}_{F T} \widehat{t}}{\widehat{\tau}_{T}} e^{-\widehat{t}^{2} / 2 \widehat{\tau}_{T}^{2}}, \quad \widehat{k}_{F T}=\frac{\theta_{T}}{B_{T} \beta_{T}}, \quad \widehat{\tau}_{T}=\frac{B_{T} \beta_{G}}{V_{b} G_{B}^{*}},
\end{aligned}
$$

In terms of their non-dimensional variables the functions $f_{1}, f_{2}$ and $f_{3}$ are given by

$$
\begin{aligned}
& f_{1}\left(\widehat{Y}_{L}\right)=\frac{\widehat{Y}_{L}}{Y_{c}+\widehat{Y}_{L}}, \quad Y_{c}=\frac{Y_{0}}{Y_{\max }}, \\
& f_{2}\left(\widehat{Y}_{L}\right)=\frac{1-\widehat{Y}_{L}}{Y_{c}+1-\widehat{Y}_{L}}, \quad Y_{c}=\frac{Y_{0}}{Y_{\max }}, \\
& f_{3}\left(\widehat{T}_{L}\right)=\frac{\widehat{T}_{L}}{T_{c}+\widehat{T}_{L}}, \quad T_{c}=\frac{T_{0}}{T_{L}^{H}} .
\end{aligned}
$$


Using the above rescalings we obtain the following system of nondimensional equations where we have dropped the hats for convenience

$$
\begin{aligned}
& \eta \frac{d Y_{L}}{d t}=\psi_{L}\left(\beta_{1} I G_{b} f_{2}\left(Y_{L}\right)+\frac{f_{1}\left(Y_{L}\right)}{1+\delta_{G} I^{2}}\right), \\
& \eta \frac{d A_{L}}{d t}=\epsilon_{g a} \theta_{1} I G_{b}-\left(\frac{\theta_{3}}{1+\delta_{H} I}+\theta_{2}+\theta_{4} I\right) A_{L}+\frac{\theta_{5}}{\epsilon_{l b}} A_{b}, \\
& \eta \frac{d T_{L}}{d t}=\epsilon_{a t} \theta_{4} I A_{L}-\frac{\psi_{V} f_{3}\left(T_{L}\right)}{1+\delta_{T} I}, \\
& \frac{d G_{b}}{d t}=F_{G}(t)+\frac{f_{1}\left(Y_{L}\right)}{1+\delta_{G} I^{2}}-\frac{G_{b}}{1+\delta_{G}}+\beta_{1}\left(1-I f_{2}\left(Y_{L}\right)\right) G_{b} \\
& +\left(\beta_{0}+\theta_{1}\right)(1-I) G_{b} \\
& \frac{d A_{b}}{d t}=\psi_{a}\left(\frac{1}{1+\delta_{A} I^{2}}-\frac{A_{b}}{1+\delta_{A}}\right)+\epsilon_{l b} \theta_{2}\left(A_{L}-A_{b}\right), \\
& \frac{d T_{b}}{d t}=\psi_{t}\left(F_{T}(t)+\frac{f_{3}\left(T_{L}\right)}{1+\delta_{T} I}-\frac{T_{b}}{1+\delta_{T}}\right)+\left(\beta_{4}+\beta_{5}\right)(1-I) T_{b}, \\
& \frac{d I}{d t}=\beta_{6}(1-I)+\beta_{7}\left(A_{b}-I\right)+\beta_{8}\left(\operatorname{erf}\left(w G_{b}-\rho\right)-I \operatorname{erf}(w-\rho)\right), \\
& \alpha \frac{\mathrm{d} G_{m}}{\mathrm{~d} t}=\mu_{g}\left(G_{b}-P I G_{m}\right)-\epsilon_{g g} \beta_{0} G_{m}(1-I) \\
& -\epsilon_{y g} \beta_{2}\left(\frac{\left(1+\gamma_{p}\right)}{\left(1+\gamma_{p} P\right)} \frac{\left(1+\gamma_{y} I\right)}{\left(1+\gamma_{y}\right)} G_{m}\right)-\epsilon_{y g} \beta_{2}\left(\frac{\left(1+\gamma_{I}\right)}{\left(1+\gamma_{I} I\right)} P Y_{m}\right), \\
& \alpha \frac{\mathrm{d} A_{m}}{\mathrm{~d} t}=\mu_{a}\left(A_{b}-A_{m} P\right)-\epsilon_{t a} \beta_{4}\left(A_{b}-T_{b}\right)+\epsilon_{t a} \beta_{4} \gamma_{t}\left(A_{b}-I T_{b}\right) \\
& -\epsilon_{t g} \beta_{3}\left(\frac{\left(1+\gamma_{q}\right)}{\left(1+\gamma_{q} P\right)} \frac{\left(1+\gamma_{x} I\right)}{\left(1+\gamma_{x}\right)} A_{m}\right)-\epsilon_{t g} \beta 3\left(\frac{\left(1+\gamma_{j}\right)}{\left(1+\gamma_{j} I\right)} P T_{m}\right), \\
& \alpha \frac{\mathrm{d} Y_{m}}{\mathrm{~d} t}=\beta_{2}\left(\frac{\left(1+\gamma_{p}\right)}{\left(1+\gamma_{p} P\right)} \frac{\left(1+\gamma_{y} I\right)}{\left(1+\gamma_{y}\right)} G_{m}\right)-\beta_{2}\left(\frac{\left(1+\gamma_{I}\right)}{\left(1+\gamma_{I} I\right)} P Y_{m}\right), \\
& \alpha \frac{\mathrm{d} T_{m}}{\mathrm{~d} t}=\beta_{3}\left(\frac{\left(1+\gamma_{q}\right)}{\left(1+\gamma_{q} P\right)} \frac{\left(1+\gamma_{x} I\right)}{\left(1+\gamma_{x}\right)} A_{m}\right)-\beta_{3}\left(\frac{\left(1+\gamma_{j}\right)}{\left(1+\gamma_{j} I\right)} P T_{m}\right), \\
& \alpha \frac{\mathrm{d} P}{\mathrm{~d} t}=\mu_{p}(1-P)+\epsilon_{g p} \gamma_{g} \mu_{g} P\left(1-I G_{m}\right)+\epsilon_{a p} \gamma_{a} \mu_{a} P\left(1-A_{m}\right) .
\end{aligned}
$$

where the new dimensionless parameters are given by

$$
\begin{gathered}
\beta_{0}=\frac{k_{G} k_{G I} I^{*} G_{b}^{*}}{\beta_{G}}, \quad \beta_{1}=\frac{k_{L} I^{*} G_{b}^{*}}{\beta_{G}}, \quad \beta_{2}=\frac{k_{C P} P^{*} G_{b}^{*}}{\beta_{G} G_{m}^{*}\left(1+k_{C I} I^{*}\right)}, \quad \rho=\frac{v}{c}, \quad w=\frac{G_{b}^{*}}{c}, \\
\beta_{3}=\frac{k_{D P} P^{*} G_{b}^{*}}{\beta_{G} A_{m}^{*}\left(1+k_{D I} I^{*}\right)}, \quad \beta_{5}=\frac{k_{T A} I^{*} G_{b}^{*}}{\beta_{G}}, \quad \beta_{4}=\frac{k_{T} I^{*} G_{b}^{*}}{\beta_{G}}, \quad \beta_{6}=\frac{k_{1} G_{b}^{*}}{\beta_{G} I^{*}}, \\
\beta_{7}=\frac{k_{I A} A_{b}^{*} G_{b}^{*}}{\beta_{G} I^{*}}, \quad \beta_{8}=\frac{k_{2} G_{b}^{*}}{\beta_{G} I^{*}}, \quad \theta_{1}=\frac{k_{A L} I^{*} G_{b}^{*}}{\beta_{G}}, \quad \theta_{2}=\frac{k_{L B} G_{b}^{*}}{\beta_{G}}, \quad \theta_{3}=\frac{S_{L} G_{b}^{*}}{\beta_{G}}, \\
\theta_{4}=\frac{k_{T H} I^{*} G_{b}^{*}}{\beta_{G}}, \quad \theta_{5}=\frac{k_{B L} G_{b}^{*}}{\beta_{G}}, \quad \gamma_{Y}=k_{Y I} I^{*}, \quad \gamma_{P}=k_{Y P} I^{*}, \quad \gamma_{Q}=k_{X P} I^{*},
\end{gathered}
$$




$$
\begin{gathered}
\gamma_{I}=k_{C I} I^{*}, \quad \gamma_{J}=k_{D I} I^{*}, \quad \gamma_{X}=k_{X I} I^{*}, \quad \mu_{p}=\frac{\mu G_{b}^{*}}{\beta_{G} P^{*}}, \quad \mu_{g}=\frac{M_{G} P^{*} I^{*} G_{b}^{*}}{\beta_{G}}, \\
\mu_{a}=\frac{M_{A} P^{*} G_{b}^{*}}{\beta_{G}}, \quad \psi_{L}=\frac{G_{b}^{*}}{Y_{\max }}, \quad \psi_{a}=\frac{\beta_{A} G_{b}^{*}}{\beta_{G} A_{b}^{*}}, \quad \psi_{T}=\frac{\beta_{T} G_{b}^{*}}{\beta_{G} T_{b}^{*}}, \quad \psi_{V}=\frac{\beta_{T} G_{b}^{*}}{\beta_{G} T_{L}^{H}}, \\
\delta_{G}=k_{G L} I^{* 2}, \quad \delta_{T}=k_{T L} I^{*}, \quad \delta_{A}=k_{A A} I^{* 2}, \quad \delta_{H}=k_{A S} I^{*}, \quad \epsilon_{g a}=\frac{G_{b}^{*}}{A_{L}^{*}} \\
\epsilon_{l b}=\frac{A_{L}^{*}}{A_{b}^{*}}, \quad \epsilon_{g g}=\frac{G_{b}^{*}}{G_{m}^{*}}, \quad \epsilon_{y g}=\frac{Y_{m}^{*}}{G_{m}^{*}}, \quad \epsilon_{t a}=\frac{T_{b}^{*}}{A_{m}^{*}}, \quad \epsilon_{a t}=\frac{A_{L}^{*}}{T_{L}^{H}}, \quad \epsilon_{g p}=\frac{G_{m}^{*}}{P^{*}} \\
\epsilon_{a p}=\frac{A_{m}^{*}}{P^{*}}, \quad \epsilon_{t g}=\frac{T_{m}^{*}}{G_{m}^{*}}, \quad \alpha=\frac{V_{s}}{V_{b}}, \quad \eta=\frac{V_{l}}{V_{b}}, \quad Y_{c}=\frac{Y_{0}}{Y_{\max }}, \quad T_{c}=\frac{T_{0}}{T_{L}^{H}} .
\end{gathered}
$$

The values for the nondimensional parameters are given in table 4 .

\section{Graphs of fluxes}

In this section we plot various combinations of the governing concentration variables to illustrate the evolution of the fluxes in the model. Noting that the three functions $f_{1}, f_{2}, f_{3}$ are defined to be unity over the vast majority of their ranges, we only need to consider 10 combinations of concentrations of functions, which are plotted in Figures 10 and 11.

The flux of glucose from plasma to muscle has a component which depends on insulin according to the product $I G_{b}$, which we plot in the top left panel of Figure 10. This quantity also influences the flux from plasma glucose to liver glycogen and liver FFA, see equations (2), (7), (8), and (11). The flux from plasma TAG to adipose tissue as modelled in (3) depends on the product $I T_{b}$, which is plotted in the top central panel. This is the only case where the high fat meal induces a higher response than the balanced meal. The product $I A_{L}$ describes the rate of conversion of FFA into TAG in the liver (8)-(9).

In the muscle, both glucose and FFA are used to convert $P$ into ATP, with rates that depend on $P I G_{m}$ and $P A_{m}$ respectively, as described by equations (10), (11) and (13). These two fluxes are plotted in the lower left and lower centre plots in Figure 10. The difference between balanced and high fat meals is more pronounced in the glucose term than the FFA term. The product $P T_{m}$ determines the rate of conversion of TAG to FFA in muscle as described in equations (13) and (14), (note that in our current parameterisation, $k_{D I}=0$ ).

In the top left panel of Figure 11, we plot the rate of release of glucose into the plasma from liver glycogen, this is inhibited by insulin, which we have modelled by $\beta_{G} /\left(1+k_{G L} I^{2}\right)$ in equations (2) and (7). The top right panel shows the flow of FFA from adipose tissue to plasma, which has a similar form, but with $k_{A A}$ replacing $k_{G L}$, see equation (4). The flux from liver TAG to plasma TAG is shown in the lower left panel. This has weaker insulin dependence, being of the form $\beta_{T} /\left(1+k_{T L} I\right)$ in equations (3) and (9). The oxidation of hepatic FFA is shown in the lower right panel of Figure 11, this has the form $S_{L} A_{L} /\left(1+k_{A S} I\right)$, see equation (8). All four fluxes show significant reductions for the time that insulin is elevated, and not a great difference between the high fat mean and the balanced meal.

\section{References}

1. D.E. Kelley and L.J. Mandarino, 2000. Fuel Selection in Human Skeletal Muscle in Insulin Resistance. Diabetes 49, 677-683.

2. D.E. Kelley, 2005. Skeletal Muscle Fat Oxidation: Timing and Flexibility are Everything. The Journal of Clinical Investigation 115, 1699-1702. 
Table 4 List of nondimensional parameters, their interpretation, and values used to produce the example results.

\begin{tabular}{|c|c|c|}
\hline Parameter & Value & Description \\
\hline$\beta_{0}$ & 0.8289 & Rate of muscle glucose uptake from plasma \\
\hline$\beta_{1}$ & 0.2632 & Rate of hepatic glucose uptake from plasma glucose \\
\hline$\beta_{2}$ & 26.32 & Rate of muscle glucose - glycogen conversion \\
\hline$\beta_{3}$ & 263.16 & Rate of muscle FFA - muscle triglyceride conversion \\
\hline$\beta_{4}$ & 0.5263 & Rate of muscle triglyceride uptake from plasma \\
\hline$\beta_{5}$ & 0.0329 & Rate of uptake of triglycerides into adipose tissue \\
\hline$\beta_{6}$ & $3.669 \times 10^{5}$ & Influence of blood glucose on insulin \\
\hline$\beta_{7}$ & $2.632 \times 10^{3}$ & Influence of blood FFA on insulin \\
\hline$\beta_{8}$ & $3.872 \times 10^{5}$ & Quadratic influence of blood glucose on insulin \\
\hline$\rho$ & 1.3926 & insulin production: ratio of glucose threshold to range \\
\hline$w$ & 0.87 & ratio of steady glucose conc to range of insulin production \\
\hline$\gamma_{p}$ & 0 & $P$-inhibited conversion of glucose to glycogen \\
\hline$\gamma_{i}$ & 0 & Insulin-inhibition of glycogen conversion to glucose \\
\hline$\gamma_{y}$ & 0.05 & Insulin-activated conversion of glucose into glycogen \\
\hline$\gamma_{q}$ & 0 & $P$-inhibited conversion of muscle FFA to triglycerides \\
\hline$\gamma_{j}$ & 0 & Insulin-inhibition of muscle triglyceride conversion to FFA \\
\hline$\gamma_{x}$ & 0 & Insulin-activated conversion of muscle FFA into triglyceride \\
\hline$\mu_{p}$ & 263.16 & Rate of AMP creation \\
\hline$\mu_{g}$ & 2.6316 & Rate of glucose oxidation \\
\hline$\mu_{a}$ & 5.2632 & Rate of FFA oxidation \\
\hline$\psi_{L}$ & 0.0161 & Plasma glucose - hepatic glycogen constant \\
\hline$\psi_{a}$ & 5.79 & FFA source constant \\
\hline$\psi_{T}$ & 0.592 & Triglyceride source constant \\
\hline$\psi_{V}$ & 0.007 & Hepatic TAG secretion rate \\
\hline$\delta_{G}$ & 0.265 & Insulin-inhibition of glucose source from liver \\
\hline$\delta_{T}$ & 0.125 & Insulin-inhibition of triglyceride source from liver \\
\hline$\delta_{A}$ & 0.5 & Insulin-inhibition of FFA source from adipose \\
\hline$\delta_{H}$ & 0.005 & Insulin-inhibition of hepatic FFA oxidation \\
\hline$\epsilon_{g g}$ & 5 & Ratio of steady state plasma glucose to muscle glucose \\
\hline$\epsilon_{y g}$ & 1 & Ratio of steady state muscle glucose to muscle glycogen \\
\hline$\epsilon_{t a}$ & 1 & Ratio of steady state plasma triglycerides to muscle FFA \\
\hline$\epsilon_{a t}$ & 0.0118 & Ratio of steady state liver FFA to healthy liver triglycerides \\
\hline$\epsilon_{g p}$ & 1 & Ratio of steady state muscle glucose to muscle AMP \\
\hline$\epsilon_{a p}$ & 1 & Ratio of steady state muscle FFA to muscle AMP \\
\hline$\epsilon_{g a}$ & 5 & Ratio of steady state plasma glucose to hepatic FFA \\
\hline$\epsilon_{l b}$ & 2 & Ratio of steady state hepatic FFA to plasma FFA \\
\hline$\epsilon_{t g}$ & 1 & Ratio of steady state muscle TAG to muscle glucose \\
\hline$\gamma_{a}$ & 30 & Number of $\mathrm{P}$ molecules used when FFA is oxidised \\
\hline$\gamma_{g}$ & 60 & Number of $\mathrm{P}$ molecules used when glucose is oxidised \\
\hline$\theta_{1}$ & 0.0263 & Rate of conversion of glucose to hepatic FFA \\
\hline$\theta_{2}$ & 1.553 & Rate of release of hepatic FFA into plasma \\
\hline$\theta_{3}$ & 2.632 & Rate of oxidation of hepatic FFA \\
\hline$\theta_{4}$ & 0.434 & Rate of conversion of hepatic FFA into TAG \\
\hline$\theta_{5}$ & 8.947 & Rate of uptake of FFA from plasma into liver \\
\hline$T_{C}$ & $1.177 \times 10^{-5}$ & Ratio of minimum to typical average liver fat \\
\hline$Y_{C}$ & $3.226 \times 10^{-6}$ & Ratio of minimum to maximum liver glycogen \\
\hline$\widehat{\tau}_{G}$ & 1.41 & timescale for digestion of glucose \\
\hline$\widehat{\tau}_{T}$ & 2.24 & timescale for digestion of triglycerides \\
\hline$\widehat{k}_{F G}$ & $21.2,11.6$ & nondimensional quantity of carbohydrate ingested \\
\hline$\widehat{k}_{F T}$ & $2.91,5.82$ & nondimensional quantity of triglycerides ingested \\
\hline$\alpha$ & 6.0 & volume ratio of skeletal muscle to blood plasma \\
\hline$\eta$ & 0.36 & volume ratio of liver to blood plasma \\
\hline
\end{tabular}



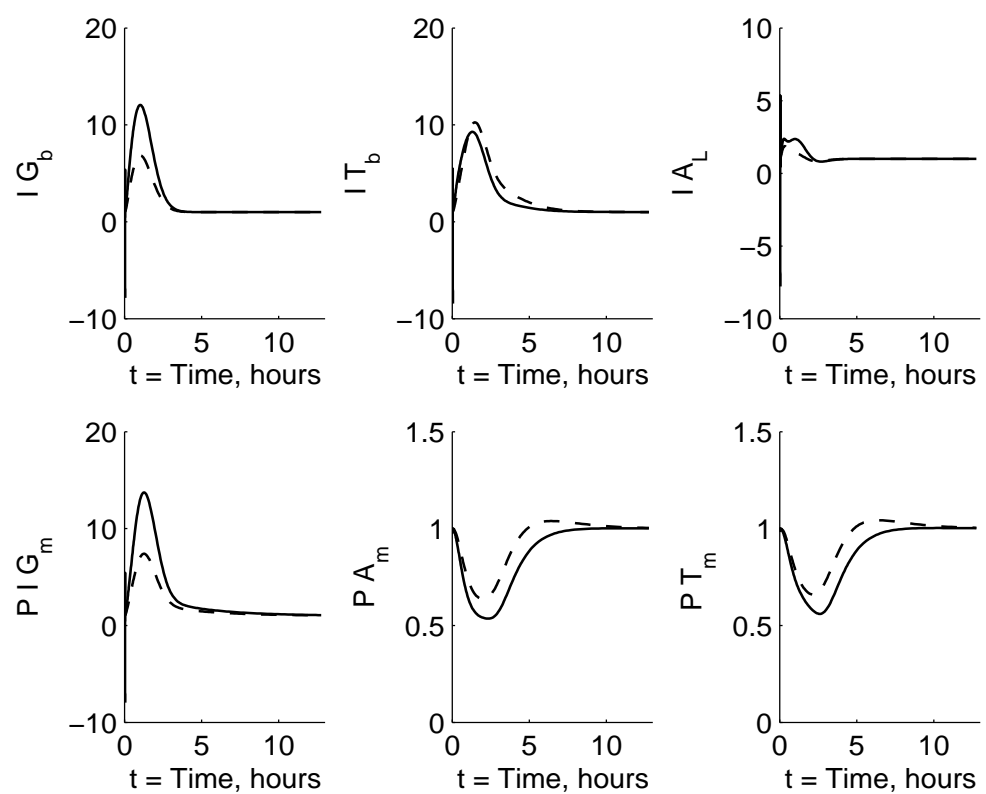

Fig. 10 Top: dimensionless fluxes involved in the model, namely the products of insulin concentration with plasma glucose (left), plasma TAG (centre) and liver FFA (right). Bottom, fluxes which involve the concentration $P$, muscle glucose with insulin (left), muscle FFA (centre) and muscle TAG (right). All graphs plotted against time in hours. Dotted lines indicate the results for the high fat meal, whilst the solid lines represent the balanced meal.

3. J.M. Olefsky, 1981. Insulin Resistance and Insulin Action: An In Vitro and In Vivo Perspective. Diabetes 30, 148-162.

4. A. Mizuno, H. Arai, M. Fukaya, M. Sato, Y.O. Hisami, E. Takeda and T. Doi, 2007. Earlyphase insulin secretion is disturbed in obese subjects with glucose intolerance. Metabolism Clinical and Experimental 56, 856-862.

5. R. Prager, P. Wallace and J.M. Olefsky, 1986. In Vivo Kinetics of Insulin Action on Peripheral Glucose Disposal and Hepatic Glucose Output in Normal and Obese Subjects. J Clin Investigation 78, 472-481.

6. P. Iozzo, K. Hallsten, V. Oikonen, K.A. Virtunen, J. Kemppainen, O. Solin, E. Ferrannini, J. Knuuti and P. Nuutila, 2003. Insulin-Mediated Hepatic Glucose Uptake Is Impaired in Type 2 Diabetes: Evidence for a Relationship with Glycemic Control. J Clin Endocrinology \& Metabolism, 88, 2055-2060.

7. A. Kotronen, , L. Juurinen, M. Tiikainen, S. Vehkavaara, H. Yki-Jrvinen, 2008. Increased Liver Fat, Impaired Insulin Clearance, and Hepatic and Adipose Tissue Insulin Resistance in Type 2 Diabetes. Gastroenterology 135, 122-130.

8. E. Phielix and M. Mensink, 2008. Type 2 Diabetes Mellitus and Skeletal Muscle Metabolic Function. Physiology \& Behaviour 94, 252-258.

9. M.A.M. den Boer, J.F.P. Berbée, P. Reiss, M. van der Valk, P.J. Voshol, F. Kuipers, L.M. Havekes, P.C.N. Rensen and J.A. Romijn, 2006. Ritover Impairs Lipoprotein LipaseMediated Lipolysis and Decreases Uptake of Fatty Acids in Adipose Tissue. Arteriosclerosis, Thrombosis and Vascular Biology 26, 124-129.

10. K.F. Peterson, S. Dufour, D.B. Savage, S. Bilz, G. Solomon, S. Yonemitsu, G.W. Cline, D. Befroy, L. Zemany, B.B. Kahn, X. Papademetris, D.L. Rothman and G.I. Shulman, 2007. The role of skeletal muscle insulin resistance in the pathogenesis of the metabolic syndrome. Proc. Natl. Accd. Sci. 104, 12587-12594.

11. G. Bock, E. Chittilapilly, R. Basu, G. Toffolo, C. Cobelli, V. Chandramouli, B.R. Landau and R.A. Rizza, 2007. Contribution of Hepatic and Extrahepatic Insulin Resistance to the Pathogenesis of Impaired Fasting Glucose. Diabetes 56, 1703-1711. 

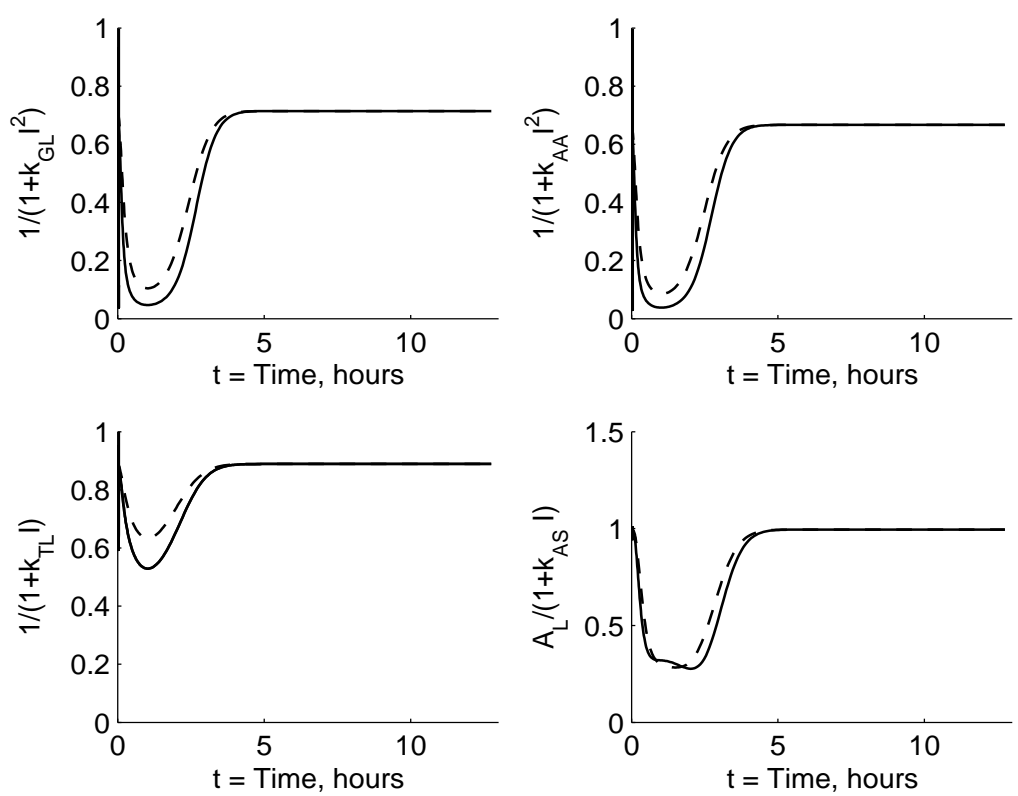

Fig. 11 Dimensionless fluxes which are suppressed by insulin, plotted again time (hours). Top left: flux from liver glucagon to plasma glucose; top right: flux of FFA into plasma from adipose tissue; lower left: flux of TAG from liver to plasma; lower right: oxidation of liver FFA. Dotted lines indicate the results for the high fat meal, whilst the solid lines represent the balanced meal.

12. R. Taylor, I. Magnusson, D.L. Rothman, G.W. Cline, A. Caumo and C. Cobelli, 1996. Direct Assessment of Liver Glycogen Storage by 13C Nuclear Magnetic Resonance Spectroscopy and Regulation of Glucose Homeostasis after a Mixed Meal in Normal Subjects. J. Clin. Invest. 97, 126-132.

13. A.S.T. Bickerton, R. Roberts, B.A. Fielding, H. Tornqvist, A.A. Blaak, A.G.M. Wagenmakers, M. Gilbert, S.M. Humphreys, F. Karpe and K.N. Frayn, 2008. Adipose tissue fatty acid metabolism in insulin-resistant men. Diabetologia 51, 1466-1474.

14. J.M. Rijkelijkhuizen, T. Doesburg, C.J. Girman, A. Mari, T. Rhodes, A. Gastaldelli, G. Nijpels and J.M. Dekker, 2009. Hepatic fat is not associated with $\beta$-cell function or postprandial free fatty acid response. Metabolism Clinical and Experimental 58, 196-203.

15. M. Adiels, J. Westerbacka, A. Soro-Paavonen, A.M. Häkkinen, S. Vehkavaara, M.J. Caslake, C. Packard, S.O. Olofsson, H. Yki-Järvinen, M.R. Taskinen and J. Borén, 2007. Acute suppression of VLDL1 secretion rate by insulin is associated with hepatic fat content and insulin resistance. Diabetologia 50, 2356-2365.

16. D.E. Harrison, M.R. Christie and D.W.R Gray, 1985. Properties of isolated human islets of Langerhans: insulin secretion, glucose oxidation and protein phosphorylation. Diabetologia 28, 99-103.

17. K.N. Frayn, 2003. Metabolic Regulation. A Human Perspective. Blackwell Publishing.

18. C. Meyer, J.M. Dostou, S.L. Welle and J.E. Gerich, 2002. Role of human liver, kidney and skeletal muscle in the postprandial glucose homeostasis. J. Physiol. Endocrinal. Metab. 282, E419-E427.

19. V. Andersen, J. Sonne, S. Sletting and A. Prip, 2000. The Volume of the Liver in Patients Correlates to Body Weight and Alcohol Consumption. Alcohol and Alcoholism 35, 531-532.

20. K.J. Acheson, Y. Schutz, T. Bessard, K. Anantharaman, J. Flatt and E. Jéquier, 1988. Glycogen storage capacity and de novo lipogenesis during massive overfeeding in man. Am. J. Clin. Nutr. 48, 240-247.

21. C. Blakemore and S. Jennett, 2001. The Oxford Companion to The Body - "Glycogen". OUP Oxford. 
22. I.M. Tolic, E. Mosekilde and J. Sturis, 2000. Modelling the insulin-glucose feedback system: The significance of pulsatile insulin secretion. Journ. Theor. Biol. 207, 361-375.

23. K. Engelborghs, V. Lemaire, J. Bélair and D. Roose, 2001 Numerical bifurcation analysis of delay differential equations arising from physiological modelling. J. Math. Biol. 42, 361-385.

24. D.L. Bennet and S.A. Gourley, 2004. Asymptotic properties of a delay differential equation model for the interaction of glucose with plasma and interstitial insulin. Applied Math. and Comp. 151, 189-207.

25. J. Li, Y. Kuang and C.C. Mason, 2006. Modelling the glucose-insulin regulatory system and ultradian insulin secretory oscillations with two explicit time delays. J. Theor. Biol. 242, $722-735$.

26. A. De Gaetano and O. Arino, 2000. Mathematical modelling of the intravenous glucose tolerance test. J. Math. Biol. 40, 136-168.

27. C. Cobelli, G. Federspil, G. Pacini, A. Salvan and C. Scandellari, 1982. An intergrated mathematical model of the dynamics of blood glucose and its hormonal control. Mathematical Biosciences 58, 27-60.

28. A.R. Sedaghat, A. Sherman and M.J. Quon, 2002. A mathematical model of metabolic insulin signaling pathways. Am. J. Physiol. Endocrinal Metab. 283, E1084-E1101.

29. Y.H. Chew, Y.L. Shia, C.T. Lee, F.A.A. Majid, L.S. Chua, M.R. Sarmidi and R.A. Aziz, 2009. Modelling of glucose regulation and insulin-signaling pathways. Molecular and Cellular Endocrinology 303, 13-24.

30. E. August, K.H. Parker and M. Barahona, 2007. A Dynamical model of lipoprotein metabolism. Bulletin of Math. Biol. 69, 1233-1254.

31. M.J. Tindall, J.A.D. Wattis, B.J. O'Malley, L. Pickersgill and K.G. Jackson, 2009. A continuum receptor model of hepatic lipoprotien metabolism. J. Theor. Biol. 257, 371384.

32. J.A.D. Wattis, B.J. O’Malley, H. Blackburn, L. Pickersgill, J. Panovska, H.M. Byrne and K.G. Jackson, 2008. Mathematical model for low density lipoprotein (LDL) endocytosis by hepatocytes. Bull. Math. Biol. 70, 2303-2333.

33. T. Pearson, J.A.D. Wattis, B.J. O'Malley, L. Pickersgill, H. Blackburn, K.G Jackson and H.M. Byrne, 2009. Mathematical modelling of competitive LDL/VLDL binding and uptake by hepatocytes. J. Math. Biol. 58, 845-880.

34. P. Vicini and M.J. Kushmerick, 2000. Cellular energetics analysis by a mathematical model of energy balance: estimation of parameters in human skeletal muscle. Am. Journ. Physiol. Cell. Physiol. 279, 213-224.

35. M.J. Lambeth and M.J. Kushmerick, 2002. A computational model for glycogenolysis in skeletal muscle. Annals of Biomed. Eng. 30, 808-827.

36. K.D. Hall, 2010. Mechanisms of metabolic fuel selection: Modelling human metabolism and body weight change. IEEE Engineering in Medicine \& Biology Magazine. 29(1), 36-41.

37. K.D. Hall, 2010. Predicting metabolic adaptation, body weight change and energy intake in humans. Am. J. Physiol. 298(3), E449-466.

38. P.N. Jordan and K.D. Hall, 2008. Dynamic Coordination of macronutrient balance during infant growth: Insights from a mathematical model. Am. J. Clin. Nutr. 87, 692-703.

39. K.D. Hall, H.L. Bain and C.C. Chow, 2007. How adaptations of substrate utilization regulate body composition. Int. J. Obesity. 31, 1378-1383.

40. K.D. Hall, 2006. Computational model of in vivo human energy metabolism during semistarvation and re-feeding. Am. J. Physiol. 291, E23-37.

41. B. Song and D.M. Thomas, 2007. Dynamics of starvation in humans. J. Math. Biol. 54, 27-43.

42. J.P. Flatt, 2004. Carbohydrate-fat interactions and obesity examined by a twocompartment computer model. Obes. Res. 12, 2013-2022.

43. V. Perival, C.C. Chow, R.N. Bergman, M. Ricks, G.L. Vega and A.E. Summer, 2008. Evaluation of quantitative models of the effect of insulin on lipolysis and glucose disposal. Am. J. Physiol. Regul. Integr. Comp. Physiol. 295, R1089-1096.

44. D.J. Klinke, 2008. Integrating epidemiological data into a mechanistic model of type 2 diabetes: validating the prevalence of virtual patients. Ann. Biomed. Eng. 36, 321-334.

45. C.E. Hallgreen, 2009. The interplay between glucose and fat metabolism: a biosimulation approach. PhD Thesis, Technical University of Denmark, Copenhagen.

46. Y. Li, T.P. Soloman, J.M. Haus, G.M. Saidel, M.E. Cabrera and J.P. Kirwan, 2010. Computational model of cellular metabolic dynamics: Effect of insulin on glucose disposal in human skeletal muscle. Am. J. Physiol. Endocrinol. Metab. 298, E1198-1209. 
47. J. Kim, G.M. Saidel and M.E. Cabrera, 2007. Multi-scale computational model of fuel homeostasis during exercise: Effect of hormonal control. Ann. Biomed. Eng. 35, 68-90

48. A Pratt, JAD Wattis, AM Salter. 2014. Mathematical modelling of hepatic lipid metabolism. submitted. 